Lars Witteck

\title{
Strafbarkeit aktiver Sterbehilfe in Deutschland - ein Trugschluss?
}

\section{Einleitung}

Die Debatte über Grenzsituationen menschlichen Lebens wird zurzeit durch die rasant fortschreitende Entwicklung der Biotechnologie dominiert. Die Möglichkeiten und Gefahren von PID, therapeutischem Klonen und Stammzellenforschung haben die Frage nach einer Zulassung aktiver Sterbehilfe in Deutschland in den Hintergrund treten lassen, ohne dass aber die Fragen über Aufnahme und Abbruch ärztlicher Maßnahmen am Beginn wie am Ende des menschlichen Lebens an Aktualität verloren hätten oder einer Lösung auch nur näher gekommen wären.

Währenddessen schreitet die internationale Rechtsentwicklung voran. Mit den Niederlanden und Belgien haben erstmals zwei europäische Nachbarländer Deutschlands ${ }^{1}$ die dort zum Teil bereits seit langem tolerierten Praktiken aktiver Sterbehilfe unter gewissen Voraussetzungen auch formell legalisiert. ${ }^{2}$

Ein solches Vorgehen scheint in Deutschland auf lange Sicht ausgeschlossen, findet sich doch in medizinrechtlichen Standardwerken zur Frage aktiver Sterbehilfe die an Deutlichkeit kaum zu übertreffende Aussage, aktive Sterbehilfe sei »nach unserer Rechtsordnung $[\ldots]$ unter keinem rechtlichen Gesichtspunkt gerechtfertigt $«{ }^{3}$ Gleichwohl wirkt es irritierend, wenn sich an derselben Stelle, lediglich durch zwei Sätze getrennt, die Aussage findet, eine »indirekte aktive Sterbehilfe « könne » [...] gerechtfertigt sein oder schon ihrem sozialen Sinngehalt nach aus dem Tatbestand der Tötungsdelikte herausfallen ${ }^{4}$, obgleich als »unbestreitbar « zugestanden wird, dass diese Form der Lebensbeendigung den Bereich des Tötungsverbotes »unmittelbar berührt ${ }^{5}$. Ist also einerseits jede Form aktiver Sterbehilfe generell und ausnahmslos verboten, gibt es andererseits aber Formen, die gleichzeitig erlaubt sind ?

1 Nach Legalisierungsversuchen einzelner Bundesstaaten in Australien und den USA, vgl. hierzu Funk, Temple International and Comparative Law Journal 2000, S. 149ff.; Datlof, Journal of Law and Health 1999-2000, S. 23ff.

2 Vgl. zu Entstehungsgeschichte und Inhalten des niederländischen »Gesetzes zur Überprüfung von Lebensbeendigung und Hilfe bei Selbsttötung « Janzen, ZRP 2001, S. 179ff. sowie Tak, ZStW 113 (2001), S. 905ff. Die belgischen Regelungen werden beschrieben bei Kintzi, DRiZ 2002, S. 256, 260 und Oduncu/Eisenmenger, MedR 2002, S. 327, 330; zust. Khorrami, MedR 2003, S. 19 ff.

3 So Ulsenheimer, in: Laufs (Hrsg.), Handbuch des Arztrechts, 3. Aufl. 2002, § 132 RN 4; ebenso Jähnke, in: Leipziger Kommentar zum StGB. 11. Aufl. 2002, vor § 211 RN 14: »Die aktive Euthanasie ist ausnahmslos unzulässig.«.

4 Ulsenheimer, aaO.

5 LK-Jähnke vor § $211 \mathrm{RN} 15$. 
Angesichts dieser Widersprüche gilt es, materiellrechtliche und rechtsethische Gesichtspunkte der Beurteilung aktiver Sterbehilfe in Deutschland zu beleuchten, um festzustellen, ob das Postulat der Rechtswidrigkeit jedweder Form aktiver Sterbehilfe in dieser Absolutheit haltbar ist. Probleme bereitet, dass das deutsche Medizinrecht weitgehend »case law « ist, dieses Rechtsgebiet kann auf eine Tradition fehlender gesetzgeberischer Entscheidung streitiger Fragen zurückblicken. ${ }^{6}$ Wenn nun auch der Bundesgerichtshof einräumt, für den Bereich der Suizidbeteiligung noch kein geschlossenes rechtliches System entwickelt zu haben ${ }^{7}$, wird verständlich, dass bei den konkreten Entscheidungen für den Betroffenen Raum ist, sich von individuellen oder standesgeprägten ethischen Einstellungen leiten zu lassen, und dass die Gerichte zu einer steten Gratwanderung zwischen den Polen des verfassungsrechtlich abgesicherten Lebensschutzes und der Respektierung der auch den Suizid umfassenden Selbstverfügungsfreiheit des Einzelnen gezwungen sind. ${ }^{8}$

Der vorliegende Beitrag versucht, ein solches rechtliches System zu liefern, ohne sich zugleich der Illusion hinzugeben, zu einer von allen Seiten akzeptierten Lösung zu gelangen. Hierzu ist der Unterschied der in die rechtliche Debatte eingebrachten theologischen, philosophischen und ethischen Standpunkte wohl zu unterschiedlich und aufgrund der dahinter stehenden Lebenseinstellungen und Vorverständnisse nur wenig kompatibel. Subjektive Erfahrungen kompensieren im Schnittfeld von Religion, Ethik, Moral und Recht das Faktum der Beschränktheit menschlicher Erkenntnisfähigkeit und beeinflussen so die Entscheidungsfindung jedes Einzelnen beträchtlich. ${ }^{9}$ Gleichwohl hat sich hinsichtlich der rechtlichen Bewertung vieler Bereiche ein Konsens herausgebildet, dessen Darstellung lohnenswert erscheint. Vor dieser Betrachtung ist jedoch eine Neubewertung einiger grundsätzlicher Differenzierungen, die die rechtliche Debatte der Sterbehilfe in Deutschland während der letzten Jahrzehnte begleitet haben, vonnöten. Diese bietet einen Blick auf die Position des Verfassers zu der wünschenswerten Regelungsform wie auch zu medizinethischen Aspekten der aktiven Sterbehilfe.

6 Dennoch sollte die Sterbehilfe-Debatte, anders als gelegentlich gefordert, nicht aus dem originär rechtsdogmatischen Diskurs ausgeschlossen werden. Die Problematik ist untrennbar verknüpft mit dem Gesichtspunkt des strafrechtlich bewährten Tötungsverbotes und kann daher nicht ausschließlich unter moraltheologischen oder -philosophischen Aspekten abgehandelt werden, vgl. auch McCall Smith, Medical Law Review 7 (1999), S. 194, 196.

7 So bereits das Gericht in der Wittig-Entscheidung von 1984 (BGHSt 32, 367, 381); beachtlich auch insoweit die Einlassung des damaligen Präsidenten Karlmann Geiß anlässlich einer Podiumsdiskussion zum 50-jährigen Bestehen des BGH im Jahre 2000, die Ergebnisse des Gerichts seien noch weniger als sonst »Erkenntnisse, die sozusagen erhoben wären über das nochmalige Reflektieren [...], ob alles, was angesprochen, angedacht und entschieden ist, ob all das Bestand hat in der weiteren Entwicklung «, in: Juristische Studiengesellschaft Karlsruhe (Hrsg.), Podiumsdiskussion: Sterbehilfe - Sterbebegleitung, Heidelberg 2001, S. 10.

8 Vgl. hierzu den Fall der Schweizer Freitodvereinigung »Exit« bei BGH JZ 2002, S. $150 \mathrm{ff}$. mit Anm. Sternberg-Lieben.

9 Hierzu auch Spranger, JZ 2002, S. 190. 


\section{Die gegenwärtige rechtliche Situation in Deutschland}

Aufgrund der fehlenden gesetzlichen Regelung von Fragen der Sterbehilfe in Deutschland ${ }^{10}$ ist eine Orientierung nur anhand von Fallgruppen möglich, die durch die höchstrichterliche Rechtsprechung und die sie begleitende Wissenschaft entwickelt wurden.

Eine Regelung hat die Materie der Sterbehilfe in den Richtlinien der Bundesärztekammer zur ärztlichen Sterbebegleitung gefunden ${ }^{11}$, bei denen es sich nach eigenem Anspruch jedoch nicht um eine Konkretisierung der strafbarkeitsbegründenden Voraussetzungen, sondern lediglich um eine Hilfestellung für den Arzt handelt, ohne diesem die rechtliche wie ethische Verantwortung in der konkreten Situation abnehmen zu können.

\section{Traditionelle und neue Differenzierungen}

Neuere Beiträge zur Diskussion der Sterbehilfe zeigen, dass überkommene Differenzierungen an Bedeutung verlieren. So scheint die Unterscheidung in Sterbehilfe durch Tun und solche durch Unterlassen in einer Zeit, da Handlungen nicht mehr in naturalistischer, sondern in normativer Weise nach ihrem sozialen Bedeutungsgehalt der Sphäre des Tuns oder Unterlassens zugeordnet werden ${ }^{12}$, an Bedeutung verloren zu haben. Gleichzeitig tritt der Gegensatz zwischen aktiver Tötung eines Schwerkranken und passivem Sterbenlassen eines solchen Patienten im Sinne einer binären Codierung von »stets verboten - mit Einschränkungen zumeist erlaubt « zurück. ${ }^{13}$

Die neue Wegscheide, an der die Verhaltenspflichten für Ärzte, Angehörige und sonstige Beteiligte entlanglaufen, scheint vielmehr in folgendem Bereich zu liegen: Das Institut der Patientenautonomie hat in den letzten Jahren eine erhebliche Aufwertung erfahren, die sich in der entscheidenden Frage äußert, ob der Betreffende in eine Lebensverkürzung eingewilligt hat oder nicht ${ }^{14}$, wobei besondere Schwierigkeiten diejenigen

10 Ein 1986 vorgelegter Entwurf einer Regelung wesentlicher, mit der Situation am Lebensende verbundener Rechtsfragen hat auf dem 56. Deutschen Juristentag zu lebhaften Diskussionen geführt, ist aber bisher von Seiten des Gesetzgebers nicht übernommen worden, vgl. Baumann u.a., Alternativentwurf eines Gesetzes über Sterbehilfe, 1986; kritisch zu den diesem Vorhaben zugrunde gelegten Prämissen Tröndle, ZStW 99 (1987), S. 25ff.

11 Abgedruckt in der neuesten Fassung in NJW 1998, S. 3406f.; zur Entstehungsgeschichte Beleites, in: Wienke/Lippert (Hrsg.), Der Wille des Menschen zwischen Leben und Sterben - Patientenverfügung und Vorsorgevollmacht, Berlin 2001, S. 65ff.

12 Vgl. etwa Frisch, Tatbestandsmäßiges Verhalten und Zurechnung des Erfolges, Heidelberg 1988, S. 134; Wessels/Beulke, Strafrecht Allgemeiner Teil, 31. Aufl. 2001, RN 702; Stree, in: Schönke/Schröder, StGB, 26. Aufl. 2001, RN 160 vor $\S 13 f f$.

13 Merkel, JZ 1996, S. 1145, 1147; Birnbacher, Rechtsphilosophische Hefte 1998, S. 75, 79ff.; Otto, Jura 1999, S. 434; Saliger, KritV 2001, S. 382, 435; sehr weitgehend aus ethischer Sicht Wils, Sterben. Zur Ethik der Euthanasie, Paderborn 1999, S. 201, 204ff.; kritisch allerdings Höfling, JuS 2000, S. 111, 113; Herbst, in: Ders. (Hrsg.), Der Mensch und sein Tod, Frankfurt/Main 2001, S. 189, 196.

14 Lipp, DRiZ 2000, S. 231, 233ff.; Hufen, NJW 2001, S. 849, 851ff.; Otto, ZfL 2002, S. 42; zurückhaltend Höfling, ZfL 2002, S. 34, 41; vgl. auch Damm, MedR 2002, S. 375ff. zu Spielarten eines »Neo-Paternalismus $«$. 
Fälle bergen, in denen der Patient zur Abgabe einer entsprechenden Erklärung nicht mehr in der Lage ist. Diese Sichtweise markiert den bedeutendsten Blickwechsel der letzten Jahrzehnte in der deutschen Diskussion um Sterbehilfe. Die Beantwortung der Frage, welche Pflichten den Arzt im Zusammenhang mit der Einstellung therapeutischer Maßnahmen im letzten Stadium des menschlichen Lebens treffen, verlagert sich mehr und mehr vom Gebiet des Strafrechts als ihrer traditionellen Heimat hin zu dem des Zivilrechts. ${ }^{15}$ Die Patientenautonomie wird als das Recht eines Patienten betrachtet, alleine zu der Entscheidung befugt zu sein, welche Art von Behandlung ein Arzt durchführen darf und welche er zu unterlassen hat. ${ }^{16}$ Mittlerweile ist die Kernfrage am Ende menschlichen Lebens nicht mehr, wann und aus welchen Gründen eine Rechtspflicht des Arztes zum Verlängern des Lebens besteht und wann er von Seiten des Rechts aus dieser Pflicht entlassen werden kann, sondern die, wann der Arzt aus Respekt vor der in dem Willen des Patienten manifestierten Autonomie verpflichtet ist, eine weitere Behandlung zu unterlassen und lebensverlängernde Maßnahmen nicht mehr vorzunehmen. ${ }^{17}$

Das Selbstbestimmungsrecht des Patienten hat zur Folge, dass der Arzt den Willen des Patienten nicht durch seinen eigenen ersetzen darf, mag dieser auch fachlich fundierter und in objektiver Hinsicht »besser « für das Wohl des Patienten sein. ${ }^{18}$ Leitbild der jüngeren Diskussion ist also nicht länger der Arzt mit seiner Pflicht zur Erhaltung des Lebens, sondern der Patient, der kraft seines grundrechtlich abgesicherten Selbstbestimmungsrechts ${ }^{19}$ die Grenzen der Therapie auch am Lebensende autonom und verbindlich bestimmen kann.

Der Wechsel von der eher die Grenzen der Autonomie in den Blick nehmenden Sichtweise des Strafrechts zu der mehr zukunftsoffenen und -gestaltenden des Zivilrechts ${ }^{20}$ hat damit zur Folge, dass das Strafrecht mit seiner traditionellen Aufgabe der

15 Halliday/Witteck, JZ 2002, S. 752, 754; Bernat, in: Wienke/Lippert (o. Fn. 11), S. 119.

16 Vgl. dazu aus jüngster Zeit nur Eberbach, in: Wienke/Lippert (o. Fn. 11), S. 11ff.; BGH JZ 2002, S. 150, 151; Kutzer, MedR 2001, S. 77; kritisch zu dem Konzept der Patientenautonomie als »vorherrschendem moralischen wie rechtlichen Leitbegriff«Eibach/Schaefer, MedR 2001, S. $21 \mathrm{ff}$.

17 Dies wurde auch von einigen Vertretern des Strafrechts bereits frühzeitig erkannt, vgl. etwa Tröndle, ZStW 99 (1987), S. 25, 44; sowie jüngstens Otto, in: Verhandlungen des 63. DJT 2000, S. K 85.

18 Uhlenbruck, ZRP 1986, S. 209, 211; OLG München, NJW 1987, S. 2940, 2943; Bottke, in: Dt. Sektion der Int. Juristenkommission (Hrsg.), Lebensverlängerung aus medizinischer und rechtlicher Sicht, Heidelberg 1995, S. 35, 95f.; Schreiber, ebenda, S. 129, 132; vgl. auch bereits BGHSt 11, 111, 114 (»Myom-Fall«).

19 Rechtsdogmatisch wird die Patientenautonomie in der deutschen Rechtswissenschaft als Anwendungsfall des Selbstbestimmungsrechts des Patienten gewertet, das seinerseits als Bestandteil einer verfassungsrechtlichen Garantie zur allgemeinen Entscheidungsfreiheit im Sinne einer selbstbestimmten Lebensgestaltung gilt. Erreicht wird diese Wertung mittels einer Zielbestimmung des Art. 2 II GG, der als Freiheitsrecht nicht lediglich den konkreten Gesundheitszustand eines Patienten schützen soll, das Grundrecht »[...] gewährleistet zuvörderst Freiheitsschutz im Bereich der leiblich-seelischen Integrität des Menschen, nicht aber beschränkt es sich auf speziellen Gesundheitsschutz.«, vgl. BVerfGE 52, 131, 174ff. Zur Debatte ausführlich Dauster, in: Wienke/Lippert (o. Fn. 11), S. 89ff.

20 Zu diesem Wechsel der Blickrichtung Taupitz, Gutachten A zum 63. Deutschen Juristentag 2000, S. A 11; Saliger, KritV 2001, S. 382, 390ff. u. pass. 
retrospektiven Verantwortungszuweisung immer weniger gefragt und in eine sekundäre Rolle der Absicherung prozeduraler Abläufe geraten ist. ${ }^{21}$ Dies erscheint für die an den entsprechenden Entscheidungsvorgängen Beteiligten von Vorteil, da sie nicht mehr ihre Verantwortung nachträglich durch ein Verfahren vor einem Strafgericht klären lassen müssen, sondern bereits vor Eintritt der kritischen Situation, wenigstens bis zu einem gewissen Grad, Leitlinien für ihr Rechtmäßigkeit ihres Verhaltens erhalten.

\section{Die anerkannten Ausnahmen von dem Verbot der aktiven Sterbehilfe}

a) Das generelle Verbot der aktiven Sterbehilfe in $\S 216 \mathrm{StGB}$

Der eingangs aufgestellten These scheint der Existenz des $§ 216$ StGB zu widersprechen. Die von dieser Vorschrift statuierte Einwilligungssperre bewahrt eine - wenn auch gegenüber den anderen Tötungsdelikten deutlich milder ausgestaltete - Strafbarkeit gerade auch dann, wenn die Tötung aus Mitleid erfolgt und der Schwerkranke ausdrücklich und ernsthaft um den Tod gebeten hat. Der Bedeutungsverlust, den die Unterscheidung zwischen aktiver und passiver Sterbehilfe in dogmatischer Hinsicht erlitten hat, lässt sich allerdings an drei Fallgruppen exemplifizieren, die zwar eine durch aktives Tun herbeigeführte Lebensverkürzung beinhalten, über deren Straflosigkeit jedoch weitgehende Einigkeit besteht.

aa) Die erste Ausnahme: Indirekte Sterbehilfe - Hilfe beim Sterben

$\mathrm{Zu}$ nennen sind zunächst die Fälle der sog. »indirekten Euthanasie«, der Abgabe von schmerzlindernden Mitteln an Schwerstkranke oder Sterbende in Dosen, die bei anderen Menschen sofort letale Wirkung zeigen würden. Tritt als Folge der Schmerzbekämpfung eine Verkürzung der Lebensdauer des Patienten ein, wird dies nicht als strafbar angesehen, sofern die Verkürzung der Lebenszeit nicht primäres Ziel des ärztlichen Handelns ist, sondern von diesem nur als Nebenfolge der erstrebten Leidensminderung hingenommen wird. ${ }^{22}$

Während über das Ergebnis der Straflosigkeit in diesen Fällen Einigkeit herrscht, ist die Begründung bisher nicht endgültig geklärt. Zum einen wird bereits ein tatbestandsmäßiges Handeln verneint, indem das Verhalten insgesamt als sozialadäquat betrachtet oder aufgrund seines Charakters als nicht gegen das Leben gerichtete, dem Leben im Sinne einer Erleichterung der Qualen ein letztes Mal dienenden Handlung nach ihrem sozialen Gesamtsinn als das Gegenstück einer »Tötungshandlung « behandelt und so im Wege der teleologischen Reduktion nicht dem Schutzbereich der $\S \S$

$21 \mathrm{Zu}$ den damit verbundenen, ebenso vielfältigen wie weitreichenden Perspektivenveränderungen instruktiv Saliger, KritV 2001, S. 382, 421f.

22 Vgl. Tröndle/Fischer, StGB, 51. Aufl. 2003, RN 17 vor § 211; Maurach/Schroeder/Maiwald, Strafrecht Besonderer Teil/1, 8. Aufl., Heidelberg 1995, § 1 RN 36, jeweils mwN; ebenso der BGH in einem 1996 ergangenen Grundsatzurteil zu dieser Frage, vgl. BGHSt 42, 301ff. In gleicher Weise argumentiert das englische House of Lords in dem dortigen leading case Airedale NHS Trust v Bland [1993], 1 All E.R. 821, wo Lord Goff ausführt: »A doctor may, when caring for a Patient who is, for example, dying of cancer, lawfully administer painkilling drugs despite the facts that he knows that an incidental effect of that application will be to abbreviate the patient's life ...«(S. 868). 
211 ff. StGB unterstellt wird ${ }^{23}$. Diesem Konzept tritt eine Ansicht entgegen, die eine Legitimation der mit der indirekten Euthanasie verbundenen Lebensverkürzung lediglich im Wege einer Abwägung für möglich hält und dem behandelnden Arzt daher die Notstandsregelung des $§ 34$ StGB zugute kommen lassen will. ${ }^{24}$ Bei der Abwägung der betroffenen Rechtsgüter sei einer Befreiung von unerträglichen Schmerzen und den sonstigen Leiden der Vorrang gegenüber der Erhaltung eines bereits erlöschenden Lebens zu geben ${ }^{25}$, da es dem Sterbenden so ermöglicht werde, seine Würde zu behalten und »als Person zu sterben, nicht aber nur noch als ein vom Schmerz beherrschtes Wesen $\ll^{26}$.

Beachtet man die strukturelle Gleichheit beider Ansätze, bleibt festzustellen, dass der Grundsatz des Verbots der aktiven Vernichtung menschlichen Lebens hier nicht aufrechterhalten wird, das Leben also nicht in jedem Fall als höchstes Rechtsgut angesehen wird. Diese Einschätzung ergibt sich zudem aus dem Gedanken des Selbstbestimmungsrechts des Patienten: Erkennt man eine Ausübung mit Blick auf die angeblich absolute Unantastbarkeit menschlichen Lebens in jenen Situationen nicht an, führt man zugleich den Gedanken der eigenverantwortlichen Gestaltung des Lebens mittels des Lebensschutzes ad absurdum, weil dieses Recht gerade in den existentiellsten Situationen seiner Ausübung gegenüber einer Fremdbestimmung zurücktreten soll. ${ }^{27}$

Unabhängig von der subjektiven Einstellung des Agierenden zu den Folgen seines Tuns handelt es sich hier um eine Form der erlaubten aktiven Sterbehilfe. Zwar wird behauptet, indirekte Euthanasie sei aufgrund der vordergründigen Absicht der Leidensminderung etwas »qualitativ anderes « als die aktive Sterbehilfe ${ }^{28}$, jedoch besteht ein so postulierter Unterschied in der Intention des Handelnden zu den Fällen der aktiven Sterbehilfe nicht. Mit dieser Differenzierung wird vielmehr der dünne Grat, auf dem indirekte und aktive Euthanasie balancieren, in einen künstlichen Gegensatz von einer ethisch hochwertigen Erleichterung eines vorbestimmten Weges auf der einen und vermeintlichem selektivem Kosten-Nutzen-Denken auf der anderen Seite verwandelt $^{29}$, der in dieser Form tatsächlich nicht besteht. Die Verkürzung des Lebens und die Todesherbeiführung sind stattdessen zwei Seiten derselben Medaille. Zum einen ist

23 Herzberg, NJW 1996, S. 3043, 3048; Hirsch, ZRP 1986, S. 239, 240; Wessels/Hettinger, Strafrecht BT/1, 26. Aufl. 2002, RN 32.

24 Schreiber, NStZ 1986, S. 337, 340f.; Kutzer, in: Eser u.a. (Hrsg.), Festschrift für H. Salger, 1995, S. 663, 670f.; Merkel, JZ 1996, S. 1145, 1148ff.; Lackner/Kühl, StGB, 24. Aufl. 2002, RN 7 vor § 211; Otto, Jura 1999, S. 434, 440f.; Roxin, in: ders./Schroth, Medizinstrafrecht, 2. Aufl. 2001, S. 93, 97. Zu anderen Ansätzen klarstellend Arzt/Weber Strafrecht Besonderer Teil, 2000, § 3 RN 7 bei FN 11.

25 Schreiber, NStZ 1986, S. 337, 341; Kutzer, ZRP 1997, S. 117, 119.

26 Otto, Jura 1999, S. 434, 441; ähnlich BGHSt 42, 301, 305 sowie Kutzer, GS Schlüchter, S. 346, 350; kritisch hierzu LK-Jähnke vor § $211 \mathrm{RN} 15$.

27 Vgl. hierzu auch Höfling, JuS 2000, S. 111, 114; Sternberg-Lieben, JZ 2002, S. 154, 155.

28 Merkel, JZ 1996, S. 1145, 1148; Oduncu/Eisenmenger, MedR 2002, S. 327, 331; vgl. auch Schreiber, NStZ 1986, S. 337, 341, der von einem »akzeptablen Bewertungsunterschied « ausgeht, ohne diesen zu spezifizieren; ders., Festschrift für E. Hanack, 1999, S. 735, 739; ähnlich LK-Jähnke vor § 211 RN 15; differenziert auch Verrel, JZ 1996, S. 224, 226.

29 So geschehen insbesondere bei Eibach, MedR 2000, S. $10 \mathrm{ff}$. 
sich auch der Arzt, dessen primäre oder einzige Absicht es ist, die Qualen seines Patienten abzukürzen, den Folgen seines Tuns bewusst und führt den Tod des ihm anvertrauten Patienten wissentlich herbei. Zum anderen kann und wird auch in Fällen der aktiven Sterbehilfe die todbringende Handlung primär der Vermeidung großer, für den Leidenden unerträglicher Schmerzen dienen und von Mitleid getragen werden. Ein tragender moralischer Unterschied zu diesen unten unter IV.1. näher behandelten Fällen ist folglich nicht auszumachen. Die Fälle der indirekten Sterbehilfe sind demnach als Ausnahme von dem strikten Verbot der willentlichen Tötung eines anderen Menschen zu qualifizieren. ${ }^{30}$ Fraglich erscheint, ob angesichts des Konsenses über die Straflosigkeit der indirekten Sterbehilfe eine gesetzliche Klarstellung erforderlich ist. $\S 214 \mathrm{a}$ des AE-Sterbehilfe erscheint gelungen, geht jedoch über die bisher gewonnenen Kenntnisse nicht hinaus.

bb) Die zweite Ausnahme: Beihilfe zum Suizid

Das Verbot der aktiven Sterbehilfe wird weiterhin durchbrochen, wenn die Tätigkeit sich als Beihilfe zum Suizid darstellt. Anders als in anderen europäischen Rechtsordnungen $^{31}$ ist diese in Deutschland unter bestimmten Voraussetzungen nicht strafbar. ${ }^{32}$

Mit der Straflosigkeit des Suizids respektiert das Strafrecht die freie Entscheidung des Suizidenten als höchstpersönliche Entscheidung, die nicht rechtlich, sondern nur ethisch zu bewerten ist. Das Strafrecht greift erst und ausschließlich dann ein, wenn die Entscheidung nicht freiverantwortlich ist, sondern der Suizident in einer Weise beeinflusst wird, die die Annahme eines Angriffs auf sein Leben von Seiten eines Dritten nahe legt. ${ }^{33}$ Hieraus ergibt sich, dass die Wurzeln der Straffreiheit der Beteiligung

30 Ungenau daher Geiß, wenn er (in: Sterbehilfe - Sterbebgleitung, S. 12) die indirekte Sterbehilfe als »Grenzfigur « zwischen erlaubter passiver und verbotener aktiver Sterbehilfe bezeichnet, es handelt sich um einen Fall der erlaubten aktiven, wenn auch nicht primär intendierten, Sterbehilfe! Ethisch ist die Erlaubtheit dieses Vorgehens zu rechtfertigen, wenn bedacht wird, dass das Handlungsziel der indirekten Sterbehilfe nicht bloß der Tod, sondern vor allem die Bewahrung der Menschenwürde des Sterbenden ist, vgl. dazu Beckmann, ZME 44 (1998), S. 143, 151.

31 So ist etwa in Großbritannien der assisted suicide ein Verbrechen, das mit einer Gefängnisstrafe von bis zu 14 Jahren geahndet werden kann. Lehrreich zu den damit verbundenen Fragen ist der Fall der gelähmten Diane Pretty, deren Begehren, ihrem Mann Straflosigkeit für sein geplantes Mitwirken bei der Beendigung ihres Lebens zusichern zu lassen, von Seiten des obersten englischen Gerichts, des House of Lords, abgelehnt wurde. Am 29.4. 2002 bestätigte der EGMR diese Rechtsprechung, vgl. Case of Pretty v. The United Kingdom (Application no. 2346/02).

$32 \mathrm{Zu}$ kurz greift allerdings die Aussage, die Straflosigkeit der Hilfehandlungen ergebe sich schon aufgrund der Tatbestandslosigkeit des Suizids und damit aus dem Fehlen einer im deutschen Beteiligungssystem zur Teilnahme erforderlichen Haupttat, so zuletzt Ulsenheimer, in: Laufs (o. FN 3), § 149 RN 5 und Achenbach, Jura 2002, S. 542, 543, in diesem positivistisch-formalen Argument liegt nicht der Kern des Problems begründet.

$33 \mathrm{Sch} / \mathrm{Sch}-$ Eser, RN 36 vor $\S \S 211 \mathrm{ff}$. Streitig ist allerdings der Maßstab der Eigenverantwortlichkeit des Opferhandelns, hier stehen sich eine strenge Verantwortlichkeitslösung und eine an die »Ernstlichkeit« in $\S 216$ StGB anknüpfende Einwilligungslösung gegenüber, vgl. zum Streitstand statt aller Gropp, in: Pohlmeier/Schöch/Venzlaff, Suizid zwischen Medizin und Recht, 1996, S. 26ff.; Bottke; GA 1983, S. 22, 31f.; Jakobs, Strafrecht AT, 2. Aufl. 1993, 21/97f. 
an einem Suizid nicht im Bereich der Abgrenzung zwischen Täterschaft und Teilnahme, sondern im Zusammenhang der Abschichtung von Verantwortungsbereichen unter Beachtung des Prinzips der Eigenverantwortlichkeit begründet liegen. Dies schließt die objektive Zurechnung des Todes des Opfers zu den Handlungen des Außenstehenden aus, wenn jener nicht das Geschehen in einem höheren Maße beherrscht als das Opfer selbst. ${ }^{34}$

Die Abgrenzung zwischen erlaubter Hilfe zum Selbstmord und verbotener Fremdtötung ist anhand des Konzepts der Tatherrschaft vorzunehmen. Eine die Strafbarkeit des Beteiligten ausschließende Selbsttötung liegt demnach vor, wenn das Opfer über den letzten, nicht mehr reversiblen Akt selbst, allein und freiwillig entscheidet und die Durchführung dieses Akts ausschließlich in seinen Händen liegt. ${ }^{35}$ Vertraut das Opfer dagegen das $\mathrm{Ob}$ « und »Wie« dieses über die Schwelle des Todes führenden Akts einem anderen an, ist dieser nach $\S 216$ StGB strafbar. Diese Argumentation fußt auf dem Zweck des $§ 216$ StGB und damit ebenfalls in hohem Maße auf dem Eigenverantwortlichkeitsprinzip. Strafgrund des § 216 StGB ist die prinzipielle Gewährleistung der Unantastbarkeit menschlichen Lebens und der Schutz des Lebensmüden vor einer Verwirklichung seines Todesverlangens durch Dritte. ${ }^{36}$ Nur ein eigenverantwortliches, selbstbestimmtes Handeln des Sterbewilligen selbst kann demnach eine Strafbarkeit des anderen ausschließen. Indem § $216 \mathrm{StGB}$ dem Sterbewilligen die Verantwortung für die Tragweite und die Unabänderlichkeit seines Verlangens selbst auferlegen will, verbietet er es anderen Personen, ihm diese letzte Entscheidung abzunehmen und sich damit - sei es auch aus achtenswerten oder verständlichen Motiven - zum Herrn über die Existenz des Lebensunwilligen zu machen. ${ }^{37}$

Festzuhalten bleibt auch an dieser Stelle, dass das Gesetz aktive Handlungen eines Dritten, die kausal für den Tod eines anderen werden, billigt und damit einen weiteren Weg der aktiven Sterbehilfe eröffnet. ${ }^{38}$ Dies erscheint zumindest in den Fällen berechtigt, in denen ein grundsätzlicher wertmäßiger Unterschied zwischen aktiver Tötung auf Verlangen und aktiver Beihilfe zum Suizid nicht besteht, etwa wenn der Helfende nur deshalb Täter i.S.d. § 216 StGB ist, weil der Leidende den eigenverantwortlich gefassten Todeswunsch nicht selbst erfüllen kann. ${ }^{39}$ Ein solches Tun erscheint nicht strafwürdiger als die Veranlassung einer freiverantwortlichen, aber lebensmüden Person zum Suizid mit dem Hintergedanken, sich dieser Person zu entledigen. ${ }^{40}$

34 Neumann, JA 1987, S. 245, 248f.; Brandts/Schlehofer, JZ 1987, S. 442, 443; Frisch, Tatbestandsmäßiges Verhalten und Zurechnung des Erfolges, S. 159; BGH, JZ 2002, S. $150,153$.

35 Sch/Sch-Eser § 216RN 11; Roxin, Täterschaft und Tatherrschaft, 7. Aufl. 1999, S. 568f.; Hirsch, ZRP 1986, S. 239, 241; Otto, in: Jescheck (Hrsg.), Festschrift für H. Tröndle, 1989, S. 162f.; Lackner/Kühl § 216 RN 3.

36 Kutzer, NStZ 1994, S. 110, 114.

37 Otto, Strafrecht BT, 6. Aufl. 2002, § 6 RN 2.

38 In diesem Sinne ebenfalls Achenbach, Jura 2002, S. 542, 547.

39 Wie hier Arthur Kaufmann, in: Schünemann u.a. (Hrsg.), Festschrift für C. Roxin, 2001, S. $841,851$.

40 Arthur Kaufmann, FS Roxin, aaO. 
cc) Die dritte Ausnahme: Das Abschalten lebenserhaltender Apparate

Eine weitere Ausnahme von der Strafbarkeit aktiver Euthanasie stellt der Therapieabbruch durch das Abschalten lebenserhaltender medizinischer Apparate dar. Obgleich das Drücken des Abschaltknopfes ein aktives Tun darstellt, soll es nicht unter die Fallgruppe der aktiven Sterbehilfe fallen, sondern nach seinem sozialen Sinngehalt als passive Sterbehilfe gewertet werden. ${ }^{41}$

Den leading case dieser Fallgruppe bildet ein 1986 vom LG Ravensburg erlassenes Urteil, in dem der Ehemann einer aufgrund einer Rückenmarkserkrankung nahezu vollständig gelähmten und ausdrücklich eine künstliche Beatmung ablehnenden, um den Tod flehenden Frau deren Wunsch vollzog und in einem unbeobachteten Moment den Respirator abschaltete, woraufhin seine Frau innerhalb von einer Stunde verstarb. ${ }^{42}$ Das Ergebnis der Straflosigkeit des Ehemanns soll hier nicht in Frage gestellt werden: Bei der künstlichen Beatmung der Patientin handelte es sich um eine gegen ihren Willen aufgedrängte und damit rechtswidrige Behandlung. Ihr Mann hat nur ihrem Selbstbestimmungsrecht zur Durchsetzung verholfen und kann daher nicht dem Anwendungsbereich einer Strafnorm bzw. dem Verdikt eines rechtswidrigen Verhaltens unterfallen.

Dennoch: Mag es auch gute Gründe geben, den aus zutiefst humanen Motiven handelnden Angehörigen oder Arzt, der durch aktives Tun lebenserhaltende Maßnahmen im Einklang mit dem Willen des Patienten beendet, nicht anders zu behandeln als den, der die Aufnahme solcher Maßnahmen erst gar nicht veranlasst, erscheint die Gleichsetzung einer Lebensbeendigung durch Tun mit der passiven Sterbehilfe doch als Ausweichen vor der klaren Stellungnahme, dass es sich um eine weitere Ausnahme von dem Verbot der aktiven Sterbehilfe handelt. Die Umdeutung des Verhaltens des das Beatmungsgerät Abschaltenden in ein »Unterlassen durch Tun ${ }^{43}$ stellt einen »Trick « der Rechtspraxis dar, um Straffreiheit gewähren zu können, indem der Rechtsunterworfene mittels der Respektierung des Selbstbestimmungsrechts aus einer etwa bestehenden Garantenstellung entlassen wird. Zu diesem zirkulären Vorgehen ist der Rechtsanwender gezwungen, da eine Deutung der Handlung als aktives Verhalten zur Strafbarkeit aus $\S 216$ StGB führen würde und der Gesetzgeber sich bisher nicht entschließen konnte, auch das aktive Verhalten wenigstens unter bestimmten Gesichtspunkten aus der Strafdrohung herauszunehmen. ${ }^{44}$

41 LG Ravensburg, NStZ 1987, S. 229; Verrel, JR 1999, S. 5, 6; anders Jescheck/Weigend, Strafrecht Allgemeiner Teil, 5. Aufl., Berlin 1996, § 58 II 1; Otto, Jura 1999, S. 434, 438.

42 LG Ravensburg, NStZ 1987, S. 229 mit Bespr Roxin, NStZ 1987, S. $345 f f$.

43 So Roxin, NStZ 1987, S. 345, 349.

44 Wie hier auch Otto, Strafrecht BT, § 6 RN 28; LK-Jähnke vor § 211 RN 18; Scheffler, in: Joerden (Hrsg.), Der Mensch und seine Behandlung in der Medizin, Berlin 1999, S. 249, 272, sowie Gropp, in Duttge u.a. (Hrsg.), Gedächtnisschrift für E. Schlüchter, 2003, S. 173, $184 f$. 


\section{Die passive Sterbehilfe}

Bei der passiven Sterbehilfe stellt sich die Frage, inwieweit es Angehörigen und Ärzten erlaubt ist, ein dem Ende sich zuneigendes Leben nicht mit allen ihnen zur Verfügung stehenden Mitteln zu verlängern, also eine lebensverlängernde Behandlung abzubrechen oder gar nicht erst aufzunehmen. Aus dem Begriff der »passiven« Sterbehilfe soll jedoch nicht die Legitimation völliger Passivität am Krankenbett abgeleitet werden. Unabhängig von der Einstellung der Bemühungen um eine Lebensverlängerung besteht eine Pflicht zur Linderung akuter Leiden und zur Verringerung der mit dem Sterbeprozess verbundenen Qualen. ${ }^{45}$ Es handelt sich um eine Änderung des Therapieziels in Richtung einer palliativmedizinischen Behandlung, bei der die elementare Grundversorgung des Patienten gewahrt bleibt. Die entscheidenden Fragen in diesem Bereich drehen sich folglich um die Reichweite der ärztlichen Pflicht zur Lebenserhaltung und ihrer Abhängigkeit von den Kriterien des Einsetzens des Sterbeprozesses sowie der Einwilligung des Patienten.

\section{a) Einverständlicher und einseitiger Behandlungsabbruch beim Sterbenden}

Die Fälle des einverständlichen Behandlungsabbruchs bezeichnen Situationen, in denen der Patient in den Abbruch einer lebensverlängernden ärztlichen Behandlung ausdrücklich oder mutmaßlich eingewilligt bzw. diesen explizit gefordert hat. Der einseitige Behandlungsabbruch dagegen bezeichnet Situationen, in denen das ärztliche Verhalten weder von einer tatsächlichen noch von einer mutmaßlichen Einwilligung des Patienten gedeckt ist. Beide Formen werden als zulässig angesehen, wenn das Grundleiden des Kranken einen irreversiblen Zustand erreicht, der Sterbeprozess bereits eingesetzt hat und der Eintritt des Todes in kurzer Zeit zu erwarten ist. ${ }^{46}$ Der Arzt ist nicht verpflichtet, erlöschendes Leben um jeden Preis zu verlängern, seine Handlungspflicht wird durch Unumkehrbarkeit und Unmittelbarkeit der Todesnähe begrenzt. ${ }^{47}$

Intensivmedizinische Betreuung wird demnach als unzulässig, ihre Beendigung als nicht strafwürdig angesehen, wenn ihre Fortführung dem ausdrücklichen oder mutmaßlichen Willen des Sterbenden widerspricht. ${ }^{48}$ Das Selbstbestimmungsrecht des Patienten schließt auch die Selbstbestimmung zum Tode ein, der vom urteilsfähigen Patienten in der Sterbephase ausgesprochene Verzicht auf weitere Behandlung wird daher für den Arzt als bindend angesehen. ${ }^{49}$ In der deutschen Strafrechtswissenschaft wird allerdings Wert darauf gelegt, dass die Einwilligung in den Behandlungsstopp

45 Ebenso Schreiber, in: FS Hanack, 1999, S. 735, 740; vgl. auch die Richtlinien der BÄK zur Sterbebegleitung, NJW 1998, S. 3406f., die von einer »Änderung des Therapieziels« in Richtung palliativer Betreuung sprechen.

$46 \mathrm{Vgl}$. Sch/Sch-Eser, RN 29f. vor §§ $211 \mathrm{ff}$.

47 Wolfslast, in: Kreuzer u.a. (Hrsg.), Ehrengabe für A.-E. Brauneck, Mönchengladbach 1999, S. 473, 478.

48 BGHSt 37, 376, 378; Hirsch, in: Küper (Hrsg.), Festschrift für K. Lackner, 1987, S. 597, $601 \mathrm{f}$.

49 OLG München, NJW 1987, S. 2940, 2943; Deutsch, AcP 192 (1992), S. 161, 171; Eberbach, in: Wienke/Lippert (o. Fn. 11), S. 11, 13; Hufen, NJW 2001, S. 849, 851f.; Ulsenheimer, in: Laufs (o. Fn. 3), § 132 RN 32. 
zur Begründung der Straflosigkeit nicht ausreichen könne, da aufgrund der Existenz des $§ 216$ StGB Unterschiede in der Disponibilität des Rechtsguts der körperlichen Unversehrtheit und dem des Lebens bestünden. ${ }^{50}$ Geht man jedoch richtigerweise davon aus, dass die Rechtfertigungssperre des $\S 216$ StGB auf aktive Tötungen beschränkt ist ${ }^{51}$, wird man die daraus zu entnehmende Entlassung des Arztes aus seinem Garantenverhältnis aufgrund des Durchgreifens der Patientenautonomie am Lebensende bejahen können und so einem richtig verstandenen Gebot der Achtung des Lebens, das einen würdevollen Prozess des Sterbens beinhaltet, gerecht werden. Dies wird auch von der Rechtsprechung anerkannt, die darauf abhebt, dass der Arzt im Fall des entgegenstehenden Willens des Patienten nicht berechtigt ist, verlöschendes Leben zu erhalten. Am Ende des Lebens sei eine Orientierung nicht an den technischen Möglichkeiten, sondern an der Achtung des Lebens und der Menschenwürde unter Berücksichtigung des Selbstbestimmungsrechts des Patienten geboten. ${ }^{52}$

Der Wille des Patienten bindet den Arzt auch in den Fällen des einseitigen Behandlungsabbruchs, jedoch ergibt sich aus dem Selbstbestimmungsrecht des Patienten kein Anspruch auf die Durchführung einer medizinisch sinnlos gewordenen Therapie. Der Arzt kann eine eigenverantwortliche Entscheidung über Fortsetzung oder Abbruch der lebenserhaltenden Maßnahmen treffen. Die Einstellung der Maßnahmen wird als zulässig erachtet, wenn es ihm darum geht, nicht bloß den agonalen Zustand des Sterbenden in die Länge zu ziehen, sondern im Sinne einer »Hilfe beim Sterben « dem Patienten ein menschenwürdiges Sterben zu gewähren und ihn nicht lediglich zum Erleiden weiterer Qualen immer wieder von der Schwelle des Todes zurückzieht. ${ }^{53}$

Die Frage einer Rechtfertigung durch eine ausdrückliche oder mutmaßliche Einwilligung des Sterbenden in Ausübung seines Selbstbestimmungsrechts tritt hier zurück. Aufgrund der normativen Unmöglichkeit bzw. Unzumutbarkeit weiterer ärztlicher Bemühungen wird schon das tatbestandliche Vorliegen einer Tötung verneint. ${ }^{54}$ Dies muss auch dann gelten, wenn der Arzt von der Seite des Patienten oder Angehöriger zur Aufrechterhaltung aus medizinischer und ethischer Sicht nutzloser und bloß die Qualen verlängernder Maßnahmen gedrängt wird. ${ }^{55}$

\section{b) Behandlungsabbruch vor Beginn des Sterbeprozesses}

Große Probleme bereitet der Behandlungsabbruch allerdings, wenn der Verlauf des Grundleidens zwar in nicht umkehrbarer Weise auf den Tod zusteuert, der Sterbepro-

$50 \mathrm{Vgl}$. Sch/Sch-Eser, RN 28 vor $\S \S 211 \mathrm{ff}$.

51 So bereits Kreuzer, Ärztliche Hilfsleistungspflicht bei Unglücksfällen im Rahmen des § 330c StGB, 1965, S. 66 bei FN 257; ebenso LK-Jähnke § 216 RN 9; Wessels/Hettinger, Strafrecht BT/1 RN 162; and. BGHSt 13, 166ff.; 32, 367ff.

52 Vgl. BGHSt 32, 367, 380; 37, 376, 378; zustimmend Tröndle/Fischer, RN 23 vor § 211; vgl. auch Schreiber, FS Hanack, S. 735, 737f. sowie Hirsch, ZRP 1986, S. 239, 240.

53 Hirsch, FS Lackner, S. 597, 602f.; Tröndle/Fischer, RN 18 vor § 211.

54 Wolfslast, Brauneck-EG, S. 473, 478; Sch/Sch-Eser, RN 29 vor $\S \S 211 \mathrm{ff}$;; Tröndle/ Fischer, RN 18 vor $\S 211$.

55 Ebenso Bottke, in: Lebensverlängerung (o. Fn. 18), S. 35, 121; vgl. auch § 214 I Nr.4 des AE-Sterbehilfe. 
zess aber noch nicht eingesetzt hat. Ist der Patient in der Lage, seinen Willen zu äußern, bestehen aufgrund der ärztlichen Pflicht zur Respektierung der Patientenautonomie keine größeren Probleme: Der Patient darf sich jeglichen, auch jeden lebensverlängernden, Eingriff verbitten. ${ }^{56}$ Wesentlich schwieriger stellen sich jene Fälle dar, in denen der Patient nicht länger in der Lage ist, seinen Willen kundzutun.

aa) Die vermeintliche Unbeachtlichkeit des Patientenwillens bei Suizidpatienten - Fall Wittig

Die Debatte über diese Fälle wurde bis in die 90'er Jahre hinein von einer Ausnahme bestimmt, die der BGH 1984 in der $»$ Wittig-Entscheidung ${ }^{57}{ }^{57}$ hinsichtlich der Beachtlichkeit des Sterbewillens von Suizidenten gemacht hat. Der BGH entschied, dass die Garantenstellung des Arztes für seinen suizidalen Patienten jedenfalls mit Eintritt der Bewusstlosigkeit, mit der dem Arzt die alleinige Tatherrschaft zufalle, nicht mehr von dem eigenverantwortlichen Todeswunsch des Patienten überlagert werde, den Arzt also in diesem Moment die Pflicht treffe, alles zur Rettung des Patienten Erforderliche zu tun. ${ }^{58}$ Grundsätzlich sei es für den Arzt unzulässig, sich dem »Todeswunsch des Suizidenten zu beugen«. Begründet wurde diese Weisung vor allem mit dem Umstand, dass Suizidenten oftmals nicht in voll verantwortlichem Zustand handelten und nach Erkenntnissen der neueren Suizidforschung ein wesentlicher Teil der Selbstmordversuche in Deutschland sog. Appellselbstmorde seien, bei denen nicht der Todeswunsch, sondern ein »verzweifelter Schrei nach menschlichem Beistand « das beherrschende Motiv sei. ${ }^{59}$ Dr. Wittig, der als Hausarzt seine Patientin nach einem Suizidversuch bewusstlos aufgefunden und in Respektierung ihres Sterbewillens eine Einlieferung in ein Krankenhaus nicht veranlasst hatte, wurde lediglich mit Blick auf seine ärztliche Gewissensentscheidung angesichts der im Fall der Rettung zu erwartenden schweren Schädigungen der Patientin freigesprochen. Diese Entscheidung habe sich an den ärztlichen Standesrichtlinien zu orientieren; der Sterbewunsch des Patienten sei lediglich einer unter verschiedenen Faktoren im Rahmen dieser Abwägung. ${ }^{60}$

Entscheidend für die einhellige Ablehnung des Urteils in der deutschen Strafrechtswissenschaft ${ }^{61}$ scheinen weniger dogmatische Fehlleistungen wie die Umfunktionierung der Tatherrschaft zu einem strafbegründenden anstatt eines täterschaftsbegründenden Merkmals zu sein. Beiseite gelassen sei hier auch die absurde Konsequenz, dass ein Beteiligter hiernach alles tun darf, um dem Selbstmörder zu einem Suizid zu verhelfen, solange dieser noch bei Bewusstsein ist; dann aber alles tun muss, um den Erfolg zu verhindern. Bedeutsam ist vielmehr, dass der Wille eines zum Sterben entschlossenen Menschen für unbeachtlich erklärt und stattdessen dem Arzt ein Abwägungsermessen eingeräumt wird, innerhalb dessen das Selbstbestimmungsrecht des

56 Vgl. statt aller Kutzer, NStZ 1994, S. 110, 113; Verrel, JR 1999, S. 5, 7.

57 BGHSt 32, 367ff.

58 BGHSt 32, 367, 374.

59 BGHSt 32, 367, 376.

60 BGHSt 32, 367, 377ff.

61 Eser, MedR 1985, S. 6ff.; Gropp, NStZ 1985, S. 97ff.; Schmitt, JZ 1984, S. 866ff.; vgl. auch den Beschluss des 56. DJT von 1986, Verhandlungen Band II, M 194. 
Patienten nur noch einen der zu berücksichtigenden Faktoren darstellt. ${ }^{62}$ Hierdurch wird der Charakter des Selbstbestimmungsrechts ins Absurde gekehrt, übrig bleibt nur ein Fremdbestimmungsrecht, was den Sterbewillen auch todkranker Patienten zu einem disponiblen Objekt herabwürdigt. ${ }^{63}$

Zuzugeben ist, dass die Erkenntnisse der modernen Suizidforschung darauf hindeuten, dass nur eine Minderheit der in Deutschland unternommenen Selbstmordversuche wirklich freiverantwortlich ist, und dass die Rechtspraxis bisweilen zu einer vorschnellen Bejahung der Freiverantwortlichkeit kommt. ${ }^{64}$ Dennoch erscheint das scharfe Schwert des Strafrechts auch bei Suizidenten nicht als das geeignete Mittel, um die Unterstützung von solch existentiellen Entscheidungen zu pönalisieren, wenn diese wenigstens subjektiv als freiverantwortlich erscheinen. ${ }^{65}$ Dem Hinweis Kutzers, die Entscheidung schränke lediglich die Beachtlichkeit des Sterbewillens des Suizidpatienten, nicht die des anders zu beurteilenden »Normalpatienten « ein ${ }^{66}$, ist bereits durch das praktische Ineinandergreifen beider Gruppen die Grundlage entzogen ${ }^{67}$. Das Urteil des BGH gab daher durchaus Anlass zu Bedenken im Hinblick auf eine Respektierung des Patientenwillens als Richtlinie bei der Einstellung lebenserhaltender Maßnahmen vor Beginn des Sterbeprozesses.

\section{bb) Der Fortschritt der Rechtsprechung - Kemptener Tee-Fall}

Eine begrüßenswerte Klarstellung hat die Kemptener-Tee-Entscheidung des BGH von $1994^{68}$ gebracht. Sie lieferte Leitlinien für den Behandlungsabbruch bei Schwerkranken mit infauster Prognose, die nicht in der Lage sind, ihren aktuellen Willen kundzutun. Dies betrifft insbesondere Patienten, die sich in einem apallischen Syndrom (Wachkoma) befinden, bei denen also die Großhirnrinde, das Pallum, ausfällt, während das Stammhirn noch erhalten ist, was nach heutigem Wissensstand eine Rückkehr zu einem personalen Dasein kaum möglich macht. ${ }^{69}$

Der BGH entschied, dass im Fall eines unheilbar erkrankten, nicht mehr entscheidungsfähigen Patienten der Abbruch einer ärztlichen Behandlung oder Maßnahme ausnahmsweise auch dann zulässig sein kann, wenn bei diesem Patienten der Sterbevorgang noch nicht eingesetzt hat. Entscheidend sei der mutmaßliche Wille des Kranken, der nach den Maßstäben der mutmaßlichen Einwilligung unter Einbeziehung von

62 Vgl. Eser, MedR 1985, S. 6, 11f.; Gropp, NStZ 1985, S. 97, 103.

63 Vgl. Schmitt, JZ 1984, S. 866f.; ablehnend auch OLG München, NJW 1987, S. 2940, 2943.

64 Nachweise bei Kutzer, MDR 1985, S. 710, 714ff.; Sch/Sch-Eser, RN 34 vor $\S \S 211 \mathrm{ff}$. sowie neuestens bei LK-Jähnke vor § 211 RN 26.

$65 \mathrm{Sch} / \mathrm{Sch}-$ Eser, RN 34 vor $\S \S 211 \mathrm{ff}$.

66 In: MDR 1985, S. 710, $711 \mathrm{f}$.

$67 \mathrm{Vgl}$. das Beispiel eines schwerkranken und lebensmüden Patienten, der seine lebenserhaltenden Medikamente nicht mehr nimmt bei Gropp, NStZ 1985, S. 97, 103 bei FN 65 sowie dasjenige eines Suizidenten, der aus der Bewusstlosigkeit erwacht und nunmehr eine lebenserhaltende Operation verweigert bei Eser, MedR 1985, S. 6, 15; vgl. auch Verrel, JZ 1996, S. 224, 230.

68 BGHSt 40, 257ff.

69 Näher zu diesen Fallgestaltungen Benzenhöfer, Der gute Tod?, 1999, S. 194ff. mwN aus dem medizinischen Schrifttum. 
früheren mündlichen oder schriftlichen Äußerungen, religiöser Überzeugung und persönlichen Wertvorstellungen unter Zugrundelegung strenger Maßstäbe zu ermitteln sein soll. ${ }^{70}$ Erst wenn sich bei der gebotenen Prüfung konkrete Anzeichen für den Patientenwillen nicht finden ließen, so könne und müsse auf allgemeinen Wertvorstellungen zurückgegriffen werden. Dabei sei Zurückhaltung geboten; im Zweifel habe der Schutz menschlichen Lebens Vorrang vor persönlichen Überlegungen des Arztes oder einer anderen beteiligten Person. ${ }^{71}$ Der Gefahr, dass Ärzte oder Angehörige ein Leben beenden, das nach ihren eigenen Vorstellungen sinnlos, lebensunwert oder nutzlos ist, müsse entgegengewirkt werden. ${ }^{72}$ Die vor mehreren Jahren unter dem unmittelbaren Eindruck einer Fernsehsendung erfolgte Äußerung der mittlerweile nicht mehr ansprechbaren Patientin, »so wolle sie nicht enden «, biete jedenfalls keine tragfähige Grundlage für eine mutmaßliche Einwilligung zum Behandlungsabbruch. ${ }^{73}$

Das Urteil belegt, dass Straflosigkeit nach dem Grundgedanken der mutmaßlichen Einwilligung möglich ist, allerdings mit der Besonderheit, dass die Disponibilität des Rechtsguts in den Fällen der passiven Sterbehilfe nicht mehr erforderlich ist. Diese Lösung ist als Stärkung der Patientenautonomie begrüß $\mathrm{t}^{74}$, aber auch aufgrund der fehlenden aktuellen Einwilligungsfähigkeit sowie der fehlenden personalen Identität zwischen dem Inhaber der für die mutmaßliche Einwilligung zu berïcksichtigenden Interessen und dem Adressaten ihrer Auswirkungen als ungeeignet zur Lösung dieser Fälle abgelehnt worden ${ }^{75}$. Die Frage nach der Grenze des Autonomieprinzips stellt sich, wenn man bedenkt, dass es sich hier um Menschen handelt, deren äußere Hülle weitgehend intakt ist, deren frühere Persönlichkeit hingegen nicht mehr existiert. ${ }^{76}$ Die insbesondere von Merkel ${ }^{77}$ gezogene Konsequenz, jene Menschen zum Gegenstand

70 BGHSt 40, 257, 263. In der Lehre war bis dahin vorgeschlagen worden, sterbende Menschen und irreversibel bewusstlose Patienten hinsichtlich der ärztlichen Verpflichtung zur Lebenserhaltung gleichzustellen, vgl. Hirsch, FS Lackner, S. 597, 603; Schreiber, NStZ 1986, S. 337, 342; zu der Entwicklung auch Schöch, FS Hirsch, S. 693ff.

71 BGHSt 40, 257, 263.

72 BGHSt 40, 257, 260f.

73 BGHSt 40, 257, 261. Wenn in diesem Zusammenhang kritisiert wird, ein Abstellen auf den mutmaßlichen Willen sei »gefährlich«, da Vermutungen in der sensiblen letzten Lebensphase eines Menschen keinen Stellenwert haben könnten (so Dauster, in: Wienke/Lippert [o. Fn. 11], S. 89, 99), so ist dazu zu bemerken, dass es für eine strafrechtliche Haftungsfreistellung auf das Vorliegen einer Einwilligung zum Zeitpunkt der Tatbegehung ankommt. Kann eine solche wegen der Kommunikationsunfähigkeit der Patienten nicht gegeben werden, geht es darum, wie sie entscheiden würden, wenn sie dies im aktuell könnten. Es geht daher in Akutsituationen tatsächlich um den (aktuell) mutmaßlichen Willen, der jedoch anhand des (in der Vergangenheit niedergelegten) aktuellen Willens recherchiert werden muss, vgl. auch Schreiber, in: Ahrens u.a. (Hrsg.), Festschrift für E. Deutsch, 1999, S. 773, 782.

74 Verrel, JZ 1996, S. 224ff.; Bottke, in: Lebensverlängerung (o. Fn. 15), S. 35, $119 f$.

75 Merkel, ZStW 107 (1995), S. 545ff.; ders., JZ 1999, S. 502, 507; vgl. auch Schöch, FS Hirsch, S. 693, 705f.

76 Hierzu allerdings sehr kritisch Dörner, ZRP 1996, S. 93ff.

77 In: ZStW 105 (1997), S. 545, 568ff.; zu den Fragen von Autonomie und personaler Identität auch ders., JZ 1999, S. 502ff., wobei sein Beispielsfall auf S. 507 jedoch die Frage aufwirft, ob die Entscheidung über lebenserhaltende Maßnahmen davon abhängen soll, ob der Betreffende »offensichtlich ganz gerne lebt«. Dies würde auf eine von Dritten vorzunehmende, objektive Bewertung des Lebens nach seiner Qualität hinauslaufen, was inakzeptabel wäre. 
einer rein objektiven Abwägung zu machen, in der seine vor Eintritt in das apallische Syndrom geäußerten Interessen lediglich einer unter verschiedenen zu berücksichtigenden Punkten sind, erscheint aufgrund ihrer Konsequenzen für Fälle, in denen ein Anhaltspunkt für die frühere Einstellung des Patienten fehlt ${ }^{78}$, problematisch. Betrachtet man frühere Äußerungen, Verfügungen und Einstellungen des Patienten als mutmaßliche Einwilligung bzw. als Ausdruck eines mangelnden Interesses der Person an dem Erhalt der Körperhülle nach Absterben der Persönlichkeit, handelt es sich doch in jedem Fall um ein subjektives Interesse, das für den Arzt mehr als eine indizielle Wirkung haben sollte. ${ }^{79}$

cc) Zivilrichter als die entscheidende Instanz ? - Die Entscheidung des OLG Frankfurt Eine zunächst nur wenig beachtete Konsequenz des Kemptener Urteils hat sich mittlerweile zur wohl meistdiskutiertesten Frage in der deutschen Sterbehilfedebatte entwickelt, ob nämlich die Einwilligung eines Betreuers in den Abbruch der Behandlung vor Einsetzen des Sterbeprozesses zu ihrer Legitimation einer vormundschaftsgerichtlichen Genehmigung bedarf. Der BGH hat dies mittels analoger Anwendung des $\S$ 1904 BGB, der die Einwilligung eines Betreuers in eine Heilbehandlung oder einen ärztlichen Eingriff der Genehmigung des Vormundschaftsgerichts unterstellt, sofern die begründete Gefahr besteht, dass der Betreute auf Grund der Maßnahme stirbt oder einen schweren und länger dauernden gesundheitlichen Schaden erleidet, bejaht. ${ }^{80}$ 1998 hat das OLG Frankfurt im Fall einer fast 85-jährigen Patientin, die nach einem ausgedehnten Herzinfarkt in ein irreversibles Koma gefallen war und mittels einer Magensonde (PEG) ernährt wurde, die Rechtsfortbildung des BGH von zivilrechtlicher Seite bestätigt. ${ }^{81}$ Den zahlreichen Stellungnahmen zu dieser Frage ${ }^{82}$ soll an dieser Stelle keine weitere hinzugefügt werden, zumal das OLG Karlsruhe/Freiburg in dem

78 Nach Merkel, aaO., S. 573f., ist in diesen Fällen ebenso wie bei Kleinkindern oder von Geburt an geistig schwerst behinderten Menschen, die in ein apallisches Syndrom geraten, der Abbruch der Behandlung ohne weiteres zulässig.

79 Ebenso Otto, Jura 1999, S. 434, 438; vgl. auch Bernsmann, ZRP 1996, S. 87, 91 und Sternberg-Lieben, FS Lenckner, S. 349, 359. Wollte man diese These in der Terminologie der empiristisch-positivistischen Sichtweise Merkels ausdrücken, so wird die Frage, ob die »vollständige«, geistig-körperliche Identität innehabende Person über das Schicksal jenes Körpers bestimmen kann, der einmal ihren Persönlichkeitskern beinhaltete, oder auch über jene Person, die im nicht entscheidungsfähigen Zustand weiterlebt, bejaht. Die Tragfähigkeit der These zeigt sich nicht zuletzt daran, dass Merkel für diejenigen Fälle, in denen das vormalige Interesse auf Erhalt des Lebens um jeden Preis gerichtet ist, keine für ihn befriedigende Lösung findet, vgl. ZStW 105 (1997), S. 545, 574f.

80 BGHSt 40, 257, 261f.

81 OLG Frankfurt, NJW 1998, S. 2747ff. mit Anm. u.a. von Knieper, NJW 1998, S. 2720ff.; Saliger, JuS 1999, S. 16ff. und Frister, JR 1999, S. $73 f$.

82 Zustimmend etwa Coeppicus, NJW 1998, S. 3381, 3386f.; Verrel, MedR 1999, S. 547ff.; Otto, Jura 1999, S. 434, 440; Saliger, JuS 1999, S. 16ff.; Berger, JZ 2000, S. 797, 799f; Taupitz, DJT-Gutachten, S. A 88ff.; ablehnend u.a. Laufs, NJW 1998, S. 3399, 3400; LG München I, NJW 1999, S. 1788; Kutzer, MedR 2001, S. 77, 78f. (anders noch ders., NStZ 1994, S. 110, 114); Eberbach, in: Wienke/Lippert (o. Fn. 11), S. 11, $27 \mathrm{ff.}$ 
neuesten zu diesem Komplex ergangenen Urteil $^{83}$ die stetig vorgebrachten Argumente der Gegenansicht, die insbesondere eine fehlende Kompetenz des Betreuers sowie die mangelnde Vergleichbarkeit der in § 1904 I BGB geregelten Materie mit den Fällen eines Behandlungsabbruchs ins Feld führen, in exzellenter Weise entkräftet hat.

Festzustellen bleibt jedoch, dass das Gericht nicht etwa eine eigene Entscheidung über das Leben und Sterben des Kranken trifft, damit auch nicht zum »Richter über Leben und Tod « wird ${ }^{84}$, sondern lediglich eine unabhängige Kontrollinstanz zur Ermittlung des die Entscheidung leitenden Patientenwillens darstellt. ${ }^{85} \mathrm{Mag}$ auch zunächst ein gewisses Unbehagen den Gedanken begleiten, solch existentielle Entscheidungen auf ein Gericht zu verlagern, so zeigen sich bei erneuter Betrachtung die Vorteile jenes Modells: Das Vormundschaftsgericht agiert jenseits des hektischen Alltags der Intensivstationen ebenso wie von persönlicher Betroffenheit Angehöriger angesichts der Grenzsituation, in der diese sich befinden. So vermag die mehrstufige Sicherung von Ermittlung und Kontrolle der Entscheidungsgrundlage in einem formalisierten Verfahren Missbrauchsbefürchtungen eher entgegenzuwirken als dies ein Urteil eines Strafgerichts lange nach Abschluss der Ereignisse leisten kann. ${ }^{86}$ Es sollte nicht unterschätzt werden, dass mit dem hier eingeschlagenen Weg die Entscheidung über das Sterbenlassen eines Menschen aus den »Hinterzimmern« herausgezogen, der Prozess des Sterbens so gleichzeitig thematisiert und enttabuisiert wird und damit wieder mehr als normaler Bestandteil unseres Daseins angesehen werden kann.

Das OLG Frankfurt betonte in seiner Entscheidung ausdrücklich die gestiegene Bedeutung sogenannter Patientenverfügungen als Grundlage der Entscheidungsfindung. Der Streit, inwieweit diese Verfügungen für die Entscheidungsträger volle Bindungs-, nur Indiz- oder gar keine Wirkung entwickeln, und ob sie bei ihrem Vorliegen für den Arzt das Entscheidungskriterium der mutmaßlichen Einwilligung ablösen ${ }^{87}$, kann hier nicht weiter verfolgt werden. Hinzuweisen ist jedoch darauf, dass diese Schriftstücke auch im Fall ihrer Eindeutigkeit nicht alleinige Entscheidungsgrundlage sein können, dass vielmehr immer Erkundigungen über grundsätzliche Einstellungen des Patienten sowie über Anzeichen für einen etwaigen Sinneswandel eingezogen werden müssen, was sich gerade im Fall mangelnder Zeitnähe zum Eintritt des Notfalls aufdrängt. Ergeben sich keinerlei Anhaltspunkte, die gegen den ausdrücklich niedergelegten Willen zur Beendigung der lebenserhaltenden Maßnahmen sprechen, sollen die Verfügungen einen Indizwert von solcher Bedeutung entwickeln, der schlichtweg nicht mehr beiseite geschoben werden könne. ${ }^{88}$

83 OLG Karlsruhe/Freiburg, NJW 2002, S. 685ff.; vgl. auch OLG Frankfurt, NJW 2002, S. 689ff.

84 So Laufs in einer polemischen Formulierung, in: NJW 1998, S. 3399.

85 Dies betont mit wünschenswerter Klarheit Hufen, NJW 2001, S. 849, 855; vgl. auch Lipp, DRiZ 2000, S. 231, 238.

86 Ebenso Saliger, JuS 1999, S. 16, 18f.; Verrel, JR 1999, S. 6ff.; Otto, Jura 1999, S. 434, 440.

87 Vgl. hierzu Sternberg-Lieben, in: Eser u.a. (Hrsg.), Festschrift für T. Lenckner, 1998, S. 349ff.; Spickhoff, NJW 2000, S. 2977, 2301f.; Ulsenheimer, in: Laufs (o. Fn. 3), § 149 RN 13.

88 Vgl. auch die Grundsätze der BÄK zur Sterbebegleitung, in: NJW 1998, S. 3406f.; wie hier Hirsch, FS Lackner, S. 597, 604; Tröndle/Fischer, RN 22a vor § 211; Spickhoff, NJW 
Aus der Darstellung der rechtlichen Situation in Deutschland können zwei Schlussfolgerungen gezogen werden: Zum einen steht das Verbot aktiver Sterbehilfe nicht mehr wie der viel zitierte »Fels in der Brandung «, sondern ist von der rechtlichen wie auch der technischen Entwicklung in Medizin und Gesellschaft vielfach unterspült und von Ausnahmen durchsetzt. Zum anderen nähert sich die rechtliche Behandlung der erlaubten Formen passiver wie aktiver Sterbehilfe mit dem Ergebnis an, dass der (ggf. antizipierte) Wille des Patienten bei der Entscheidung über die Rechtmäßigkeit eines Tuns immer größere Bedeutung erlangt. Aufgrund dieser Entwicklungen wird zunehmend die Frage gestellt, ob das strafbewehrte Verbot der aktiven und willentlichen Tötung eines anderen Menschen zum Zwecke der Verkürzung seines Leidens in der heutigen Form auch dann aufrecht erhalten werden sollte, wenn die Tötung dem ausdrücklichen und ernsthaften Willen des Patienten entspricht, oder ob hier Ausnahmeregelungen angebracht sind.

\section{Die ethische Legitimation von Ausnahmeregelungen zur aktiven Sterbehilfe}

Eine solche Ausnahmeregelung hatten im Jahre 1986 die Verfasser des AE-Sterbehilfe propagiert. Nach § 216 II ihres Entwurfs kann das Gericht in Fällen der Tötung auf Verlangen von Strafe absehen, wenn die Tötung der Beendigung eines schwersten, vom Betroffenen nicht mehr zu ertragenden Leidenszustandes dient, der nicht durch andere Maßnahmen behoben oder gelindert werden kann. ${ }^{89}$ Von den materiellen Voraussetzungen her stellt dies den niederländischen Weg einer Kodifizierung dar. Jedoch erscheint dieser Weg ohne die im niederländischen Gesetz niedergelegten formalen Hürden und verfahrenstechnischen Absicherungen kaum gangbar.

Wir müssen uns erinnern: Der niederländische Weg einer Legalisierung der Sterbehilfe wurde vehement abgelehnt und als in die falsche Richtung weisender »Tabubruch « bezeichnet. ${ }^{90}$ Nimmt man diese Argumentation auf, tritt die Frage in den Vordergrund, welche anderen Wege es gibt, den Notstand am Ende des Lebens aufzulösen, der so viele ältere Menschen den Wunsch nach fremdhändiger Beendigung ihres Daseins äußern lässt. Die Debatte über die Sterbehilfe wird noch immer zu einseitig auf dem Feld des Strafrechts geführt. Die Antwort sollte stattdessen zunächst außerhalb des Rechts, auf dem Gebiet der Medizin, und sodann außerhalb des Strafrechts, auf dem Gebiet des Privatrechts, gesucht werden.

2000, S. 2977, 2301f.; Schöch, FS Hirsch, S. 693, 707; Verrel, MedR 1999, S. 547, 548f., dort auch zu den weiteren Optionen des deutschen Betreuungsrechts, vgl. aus strafrechtlicher Sicht Sternberg-Lieben, FS Lenckner, S. 349, $363 \mathrm{ff}$.

89 Vgl. Baumann u.a., AE-Sterbehilfe, S. 34ff.

90 Vgl. nur die Äußerungen der Bundesjustizministerin Däubler-Gmelin, des Vorsitzenden des Rates der EKD Kock und des Präsidenten der BÄK Hoppe bei SPIEGEL ONLINE vom 11.4.2001 unter http://www.spiegel.de /politik/deutschland/0,1518,127744,00.html; weitere Nachweise bei Saliger, KritV 2001, S. 382, 384. 


\section{Erforderlichkeit eines Blickwechsels auf dem Gebiet der Medizin}

\section{a) Der Ansatz der Palliativmedizin}

Das Ideal des Arztes als Heiler wird dort zum Problem, wo er sehen muss, dass ein Heilungserfolg nicht mehr möglich ist. In dieser Situation gilt es, einen Weg zu finden, der zwischen den beiden Polen des Erhaltens des erlöschenden Lebens um jeden Preis (als Reflex eines sinnlosen Versuchs, heilend einzugreifen) auf der einen Seite und der vorzeitigen Beendigung des Lebens (als ebenso mitleidsgetriebene wie hilflose »Trotzreaktion « auf die Erkenntnis, dass hier sämtliche Heilungsversuche vergebens sind) auf der anderen Seite liegt. Für einen Arzt mag es aufgrund seiner Ausbildung und Sozialisation mitunter schwer sein, die ihm zugewiesene Aufgabe nicht »zu Ende zu führen«, sondern durch ein schlichtes Loslassen einen für den Patienten humaneren Weg zu beschreiten, ohne dies als persönliche wie fachliche Niederlage zu empfinden. $^{91}$

Der einzuschlagende Weg kann nur der einer Begleitung sein. Es gilt, die Möglichkeiten der Palliativmedizin auszuschöpfen, die eine patientengerechte Schmerztherapie ebenso umfasst wie eine ganzheitliche therapeutische Betreuung, die nicht alleine die körperlichen Leiden des Patienten zu mildern versucht, sondern es dem Sterbenden durch menschlichen Beistand ermöglicht, seinen Lebensweg in Würde zu beschließen. Aufgabe der Palliativmedizin als Gegenstück zur Kurativmedizin ist es, den Patienten ihre körperlichen Funktionen solange als möglich zu erhalten, damit diese möglichst lange ein annähernd normales Leben führen können und nicht auf die moderne Apparatemedizin angewiesen sind..$^{92}$ Es handelt sich um einen Wechsel des Behandlungsparadigmas, es geht nicht mehr um Heilung, sondern darum, die letzte Lebensphase zu erleichtern, sie in würdiger Weise zu gestalten und damit dem Sterbevorgang das Qualvolle zu nehmen. ${ }^{93}$

Diese Forderung ist nicht neu. ${ }^{94}$ Trotz einiger Fortschritte wird in der Fachliteratur jedoch noch immer darauf hingewiesen, dass in Deutschland aufgrund der Überschätzung der Suchtgefahr und der Angst vor rechtlichen Konsequenzen aufgrund einer restriktiven Betäubungsmittelpolitik der Einsatz von Opioiden häufiger als in anderen europäischen Ländern verzögert oder sogar ganz unterlassen wird. ${ }^{95}$ In Medizinerkreisen wird eine mangelnde oder unzureichende Schmerztherapie als einer der Hauptgründe für den Wunsch nach aktiver Sterbehilfe qualifiziert, die Verbesserung

91 Vgl. hierzu auch Uhlenbruck, ZRP 1986, S. 209, 210f.; Wolfslast, Brauneck-EG, S. 473, 480.

92 Vgl. zu Geschichte und Methodik der Palliativmedizin umfassend Husebö/Klaschik, Palliativmedizin, 2. Aufl. 2000.

93 Zum derzeitigen Stand der Palliativmedizin in Deutschland Klaschik, Vortrag auf der Jahrestagung 2000 des Tumorzentrums Rheinland-Pfalz, abrufbar unter http://info.imsd.unimainz.de/TUZ/jahrestagung_00.html

$94 \mathrm{Vgl}$. bereits Kutzer, FS Salger, S. 663ff.

95 Ensink, Vortrag zum Göttinger Palliativ-Projekt SUPPORT vom 11.4.2001, abrufbar unter www.gwdg.de/ pctgoe/Seiten/Publikation/SUPPORTethics.pdf, S. 4; Sass/Zenz, MEDrubin 2001, S. 36, 41. 
dieser medizinischen Richtung mithin als Alternative zur Legalisierung der Euthanasie durch deutliche Reduzierung der diese in Anspruch nehmenden Personengruppen angesehen. ${ }^{96}$

\section{b) Der Ansatz des Hospizwesens}

Die Angst vor einem qualvollen Sterben wird dadurch verstärkt, dass der Sterbevorgang heute entgegen den Wünschen tödlich Erkrankter zumeist in der fremden und unpersönlichen Umgebung eines Krankenhauses stattfindet, das Sterben wird im Vergleich zu früheren Gebräuchen »ent-familisiert «. ${ }^{97}$ Die Rede ist von der Desozialisierung, der sich alte Menschen am Ende ihres Lebens durch die Auflösung traditioneller Familienbande gegenübersehen.

Sinnvoll erscheint daher eine weitgehende Kooperation der Palliativmedizin mit der in Deutschland aufkommenden Hospizbewegung. Indem Palliativmedizin und Hospizbewegung gestärkt werden, wird es möglich, die Angst vor einem einsamen, qualvollen Tod und den daraus resultierenden Wunsch nach Sterbehilfe in vielen Fällen zu vermeiden. Sollte dies gelingen, würde ein bedeutender Teil der Probleme, denen sich Ärzte und Juristen im Moment gegenüber sehen, entschärft werden. Viele der Wünsche nach Lebensbeendigung entspringen nämlich aus einer tiefen und existentiellen Angst, am Ende des Lebens allein zu sein und das Heft des Handelns nicht mehr in der Hand zu haben, lediglich zum Objekt eines anonymisierten Kranken- und Sterbebetriebes zu werden. ${ }^{98}$ Gelänge es, das Sterben auf diese Weise zu enttabuisieren und zu einem akzeptierten und würdevollen Teil menschlichen Lebens zu machen, wäre ein Großteil der heute Betroffenen dem Anwendungsbereich des Strafrechts entzogen, dass sich dann weder mit krampfhaften Versuchen, das Leben (u.U. gegen den Willen des Patienten) zu erhalten, oder mit dem vorschnellen und aktiven Beenden des Lebens nach dem Motto »lieber ein Ende mit Schrecken als ein Schrecken ohne Ende « befassen brauchte.

\section{Erforderlichkeit eines Blickwechsels auf dem Gebiet des Rechts}

Eine weitere Reduzierung der dem Strafrecht unterfallenden Personengruppen ist durch eine konsequente Nutzung zivilrechtlicher Möglichkeiten einer antizipierten Willensgestaltung anzustreben. ${ }^{99}$ Das Zivilrecht bietet für den Patienten mit dem sog. Patiententestament, der Betreuungsverfügung und der Vorsorgevollmacht drei Gestaltungsmöglichkeiten, seine Wünsche im Hinblick auf den Abbruch einer medizinischen Therapie für den Fall einer Unfähigkeit zur Willensäußerung darzulegen und ihre

96 Sass/Zenz, MEDrubin 2001, S. 36, 40f.; zustimmend Schreiber, in: Lebensverlängerung (o. Fn. 18), S. 129, 143 und Kutzer, GS Schlüchter, S. 346, 349; vgl. auch Ru $\beta$, EthikMed 14 (2002), S. 11, 18.

97 Vgl. Eser, JZ 1986, S. 786, 787.

98 Schreiber, in: Lebensverlängerung (o. Fn. 18), S. 129, 134f.; Tröndle, ZStW 99 (1987), S. 25, 39; Kutzer, GS Schlüchter, S. 346, 359 u. öfter.

99 Vgl. zur erforderlichen Reduktion der Rolle des Strafrechts auf dem Gebiet der Sterbehilfe Halliday/Witteck, JZ 2002, S. 752, 754 sowie Saliger, KritV 2001, S. 382, 389ff. u. pass. 
Befolgung abzusichern. Diese Rechtsfiguren ermöglichen dem Kranken eine Stärkung seines Selbstbestimmungsrechts in der finalen Phase des Lebens. Sie nehmen zugleich Handlungsdruck von Ärzten und Pflegepersonal, die ihre Entscheidungen über den Abbruch lebensverlängernder Maßnahmen nunmehr an Anweisungen von Seiten des Patienten oder rechtlich legitimierter Dritter anlehnen und so den Willen des Patienten respektieren können, ohne gleichzeitig in der Furcht zu leben, sich hierdurch strafbar zu machen.

\section{Probleme dieses Ansatzes - Schaffung begrenzter Ausnahmeregeln}

Die Frage ist nun, ob ein solches Zwischenergebnis, mit dem der Kreis potentiell von aktiver Sterbehilfe Betroffener von gleich zwei Seiten zurückgedrängt wird, zufrieden stellen kann.

Die Lösung scheint elegant: Einerseits soll über eine Verbesserung von Palliativmedizin, Schmerztherapie und Sterbekultur der Wunsch nach Sterbehilfe verringert oder gar eliminiert werden. ${ }^{100}$ Andererseits bestehen de lege lata vielfältige Möglichkeiten um ein von Patientenseite als unwürdig und elend empfundenes Leben nicht künstlich zu verlängern. Neben den durch das Zivilrecht gegebenen Gestaltungsmöglichkeiten am Lebensende gestattet auch das Strafrecht unter bestimmten Voraussetzungen den Abbruch einer ärztlichen Behandlung und sogar einige Vorgehensweisen, die eine aktive Lebensverkürzung zum Gegenstand haben. Ist es nicht schon ein Erfolg, dass das Strafrecht sich auf diejenigen Fälle zurückziehen kann, in denen missbräuchlich aktive Sterbehilfe i.S.d. $§ 216$ StGB betrieben wird ?

Bedauerlicherweise legen viele ärztliche Berichte den Schluss nahe, dass auch die modernen Möglichkeiten der Palliativmedizin nicht immer den Zustand der schwerstkranken Patienten auf ein erträgliches Maß abmildern können. ${ }^{101}$ Während die Gabe starker Schmerzmittel nicht in allen Fällen in der Lage ist, die Schmerzzustände der Patienten zu bekämpfen, hat sie zugleich den Nebeneffekt einer dauernden Bewusstseinstrübung, die den Patienten zwar schmerzfrei zurücklässt, ihn jedoch dem Ideal eines selbstbestimmten Sterbens nicht näher bringt. Weiterhin ist zu bedenken, dass es jenseits der Schmerzen Leidenszustände am Ende des Lebens gibt, denen auch mittels palliativer Betreuung nicht beizukommen ist. Der Terminus des Leidens bezeichnet einen subjektiven Zustand, der jenseits des Empfindens objektiver Beobachter liegen kann.

In gleicher Weise sind die zivilrechtlichen Vorsorgemöglichkeiten nicht kritiklos zu sehen. ${ }^{102}$ Hinsichtlich der Patiententestamente wird vorgebracht, die Anhänger einer rechtlichen Bindungswirkung berücksichtigten nicht, dass Interessen und Wünsche des einwilligungsunfähigen Patienten sich dramatisch von denen unterscheiden können, die er in gesundem und einwilligungsfähigem Zustand hatte. ${ }^{103}$ Zugleich ist der

$100 R u \beta$, EthikMed 14 (2002), S. 11, 18f.; Kutzer, GS Schlüchter, S. 346, 349; Bormann, ZME 2002, S. 29, 37; geradezu enthusiastisch Diemer, in: Herbst (o. Fn. 13), S. 255, 276.

101 Ebenso Saliger, KritV 2001, S. 382, 387 mwN.

102 Vgl. hierzu ausführlich Halliday/Witteck, JZ 2002, S. 752, $760 \mathrm{ff}$.

103 Vgl. dazu Rebecca S. Dresser, "Advance Directives, Self Determination, and Personal Identity«, in Chris Hackler u.a. (Hrsg.), Advanced Directives in Medicine, 1989, S. 160. 
den Instituten einer Stellvertretung oder Betreuung des Kranken zugrunde liegende Rekurs auf das Selbstbestimmungsrecht des Patienten missverständlich - es ist nicht der Patient selbst, der die Entscheidung trifft. Diese wird vielmehr von einer dritten Person getroffen, vermeintlich auf der Basis der Wünsche und Einstellungen des Patienten. Es stellt sich die Frage, ob dieser Ansatz in der Praxis nicht zu subjektivistisch gerät - aber auf der Seite des Bevollmächtigten. Die Annahme, Stellvertreter und Betreuer handelten ausschließlich aus altruistischen Motiven, lässt die enorme emotionale Betroffenheit von Familienmitgliedern oder sonstigen dem Patienten nahe stehenden Personen und die damit oftmals verbundene Unfähigkeit zur unvoreingenommenen Entscheidungsfindung außer acht. Selbst wenn man das Missbrauchspotential der Fälle außer acht lässt, in denen die an der Entscheidung beteiligte Person einen materiellen Vorteil vom Ableben des Patienten hat, besteht die Gefahr, dass die emotionale Reaktion dieser Person zu überwältigend ist, um den Patienten ohne weiteres sterben zu lassen oder ihn in einem Zustand weiterleben zu lassen, den sie selbst um ein vielfaches schlimmer als den Tod bewertet. Die Antwort auf die Frage danach, was der Patient wirklich will, könnte also nichts weiter als eine Reflektion der eigenen Wünsche des Auskunftsgebenden sein.

Bedenklich stimmt zudem die Existenz einer Gruppe von Personen, die von den Möglichkeiten der zivilrechtlichen Gestaltung von Entscheidungen am Lebensende nicht erreicht wird. Patiententestament, Betreuungsverfügung und Vorsorgevollmacht erlauben es einerseits den Menschen, ihrer Vorstellung vom eigenen Leben und Sterben Ausdruck zu verleihen. Sie legen ihnen mit dieser Entscheidungsmöglichkeit zugleich eine Entscheidungslast auf, die vielleicht nicht von allen als Erleichterung empfunden wird und für manchen den Gedanken an das Sterben noch schwerer macht als er für diesen ohnehin schon ist. ${ }^{104}$ Mag man diese »Pflicht zur Selbstbestimmung « noch als unabdingbare Kehrseite eines gewandelten Menschenbildes im Recht, verbunden mit gewachsenem Respekt vor der Autonomie des Einzelnen hinnehmen, so sind die Chancen, die das Recht zur Sicherung dieser Autonomie bietet, nicht für jedermann geeignet und wahrnehmbar. Diejenigen Personen, die sich zu Lebzeiten nicht über ihre Wünsche bezüglich ihres Sterbens geäußert haben, können ebenso wenig erfasst werden wie Personen, die aufgrund physischer oder psychischer Defizite nie in der Lage waren, einen Willen zu bilden.

Aus diesen Gründen macht es sich die oben dargestellte Argumentation zu einfach und muss sich den Vorwurf des Zynismus gefallen lassen. Ihre Konsequenz ist, dass gerade die schwächste und hilfloseste Gruppe von den rechtlichen Möglichkeiten der Sterbehilfe abgekoppelt wird. Das bedeutet, dass denen, die aus körperlichen Gründen auch mit der erlaubten Hilfe Dritter nicht in der Lage dazu sind, ihrem als unerträglich empfundenen Leben ein Ende zu setzen, es verwehrt wird, andere hierum zu bitten. ${ }^{105}$

104 Eibach, in: Wienke/Lippert (o. Fn. 11), S. 47ff.

105 Ebenso Hoerster, Sterbehilfe im säkularen Staat, S. 41. In den Ohren Schwerstbetroffener und ihrer Angehörigen muss es wie Hohn klingen, wenn ihnen von Bormann, ZME 2002, S. 29, 35, attestiert wird, dass obgleich »bestimmte sozial und individuell besonders wertgeschätzte Handlungsformen« nicht länger möglich seien, dies keineswegs bedeute, dass 
Damit werden aber Handlungen, die aus Mitleid oder Barmherzigkeit etwa von einem nahen Angehörigen begangen werden, der den Leidenden auf dessen Bitten hin von schwersten Qualen erlösen will, ohne Ausnahme auch dann nach § 216 StGB bestraft, wenn eine solche Tat im Einzelfall ethisch nicht missbilligenswert erscheint. ${ }^{106}$

Nimmt man diese Bedenken ernst, so sprechen die bisher in der Debatte erreichten Fortschritte nicht gegen, sondern gerade für eine Schaffung eng begrenzter Ausnahmeregelungen für Fälle, in denen die aktive Sterbehilfe nicht vom Recht verboten sein dürfte.

\section{Auseinandersetzung mit den Argumenten gegen eine Legalisierung der aktiven Sterbehilfe}

Gegen die Legalisierung aktiver Sterbehilfe in jeglicher Form werden von ethischgesellschaftspolitischer Warte die sog. »Dammbruch-Argumente« ins Feld geführt. Diese Argumente existieren in zwei Erscheinungsformen. Zum einen wird argumentiert, dass eine auch nur teilweise Legalisierung aktiver Euthanasie zur Durchführung von Formen der Sterbehilfe führen könnte, die von dem Gesetz ursprünglich nicht erfasst waren. Zum anderen könnte das Inkrafttreten des Gesetzes soziale Tabus in Bezug auf die absichtliche Tötung eines Menschen schwächen und so die Tür zu einer späteren Erlaubnis anderer Formen der Euthanasie öffnen. ${ }^{107}$

$\mathrm{Zu}$ dieser Argumentationsfigur ist zu konstatieren, dass sie trotz großer Suggestivkraft von ihrem sachlichen Gehalt her nicht überschätzt werden sollte. Diese Einschätzung haben sich ihre Vertreter durch geradezu inflationären Gebrauch der Figur selbst zuzuschreiben. Die Gefahr eines Dammbruchs oder eines Missbrauchs wird selbst dann beschworen, wenn keine materielle Neuerung eingeführt wird, sondern lediglich Entscheidungsprozesse aus der Informalität herausgezogen und einer Prozeduralisierung unterworfen werden. ${ }^{108}$ Eine derartige Zuspitzung auf schlimmstmögliche Szenarien und ein gleichzeitig fehlender Beleg für deren Eintrittswahrscheinlichkeit macht diese Argumentationsfigur zu einem mit Vorsicht zu genießenden Instrument, mit dessen Einsatz zuweilen eher eine Positionierung im Meinungskampf als ein Beitrag zum rechtlichen oder ethischen Diskurs angestrebt wird. ${ }^{109}$ Dennoch sollen an dieser Stelle

ihnen damit »die Möglichkeit genommen [sei], sich als sittliches Subjekt im Handeln auszudrücken und die verbleibende Lebensspanne als sinnvoll zu erleben«.

$106 \mathrm{Vgl}$. Baumann u.a. (Hrsg.), AE-Sterbehilfe, S. 35.

107 Vgl. Crofts, The Right of the Individual or the Common Good ?, in: Joerden/Neumann (Hrsg.), Studien zur Ethik in Ostmitteleuropa, Band 3, Frankfurt/Main 2002, S. 133, 143, 149. In normtheoretischer Hinsicht warnen die Dammbruchargumente davor, dass bei Zulassung einer bestimmten Handlung ein Prozess in Gang gesetzt wird, der zu der Gleichbehandlung sich nur minimal unterscheidender Handlungen und schließlich am Ende der schiefen Ebene zur Zulassung von Handlungen zwingt, die am Anfang von niemandem gewollt wurden. Eine Auseinandersetzung mit dieser Argumentationsfigur erfolgt bei Gukkes, Das Argument der schiefen Ebene, Stuttgart 1997. Vehement wird die Theorie des Dammbruchs vertreten bei Tröndle, ZStW 99 (1987), S. 25, 39f.

108 So geschehen bei Laufs, NJW 1998, S. 3399ff. in Auseinandersetzung mit dem erwähnten Urteil des OLG Frankfurt/Main.

109 Vgl. hierzu ausführlich Guckes, Das Argument der schiefen Ebene, S. 10 u. passim sowie Kintzi, DRiZ 2002, S. 256, 262 und Gutmann, Ethik Med 2002, S. 170, 179 f. 
zwei Argumente genannt werden, die zumindest vordergründig einiges an Überzeugungskraft beinhalten:

- Zum einen ist zu bedenken, ob die ethischen Strukturen einer Gesellschaft nicht tief greifend verändert werden, indem erlaubt wird, dass nicht nur der den Tod Suchende selbst die Herrschaft über das Geschehen hat, sondern dass Dritte seinen Tod in Vollzug setzen, das Werk selbst beherrschen und eine Fremdtötung begehen.

- Weiterhin wird auf die Gefahr hingewiesen, dass als Nebeneffekt einer schrittweisen Legalisierung aktiver Sterbehilfe Denkprozesse in Gang gesetzt werden, die auf ein Überflüssigkeitsgefühl älterer oder kranker Leute hinauslaufen. ${ }^{110}$ Speziell solche Leute könnten in depressionsähnlichen Sterbegedanken bestärkt werden, die es als Folge eines arbeitsreichen Lebens nicht gewohnt sind, nichts zu tun und anderen »zur Last zu fallen«. Diese Personen könnten nun ihr Dasein als nutzlos ansehen und aus einer Art »Anstandsgefühl« um den Tod bitten, um ihren Verwandten eine aufopfernde Pflege zu ersparen. ${ }^{111}$

Lohnenswert ist zunächst eine Auseinandersetzung mit dem zweiten Argument. Die Annahme des Missbrauchs wird hier unzulässigerweise auf Situationen der aktiven Sterbehilfe beschränkt. Man kann aufgrund der oben dargelegten Fallstricke bei zivilrechtlichen Vorsorgemöglichkeiten mit dem gleichen Recht behaupten, dass bereits de lege lata eine erhebliche Dunkelziffer von Tötungen bzw. Behandlungsabbrüchen existieren, die nicht durch den selbstbestimmten Willen des Patienten gerechtfertigt sind. ${ }^{112}$ In gleicher Weise sind Differenzierungen, die auf subjektiver Ebene über die Straffreiheit eines Verhaltens entscheiden, wie dies etwa bei der indirekten Sterbehilfe der Fall ist, in hohem Maße missbrauchsanfällig. Die in sich keineswegs konsistente gegenwärtige Rechtslage ist also bereits durch Situationen geprägt, in denen jederzeit die Gefahr eines missbräuchlichen Verhaltens besteht. Bei einer eng begrenzten Zulassung aktiver Sterbehilfe wäre daher keine wesentliche Verschlechterung zu fürchten, im Fall einer größtmöglichen Prozeduralisierung solcher Fälle ist vielmehr das Schrumpfen des heute noch erheblichen Dunkelfeldes zu erwarten.

Interessanterweise führt eine weitere Inkonsistenz dieses Arguments zu wirklich bedenklichen Fallkonstellationen: Die von den Vertretern der Dammbruchthese angeführten Beispiele älterer Leute, die aufgrund einer subjektiv empfundenen Nutzlosigkeit ihres Daseins aus dem Leben scheiden wollen und daher um eine aktive Tötung bitten, wären von der gegenwärtigen niederländischen Rechtslage, die als skandalöses Einfallstor zu einer Aufweichung des Lebensschutzes empfunden wurde, gar nicht erfasst, sofern bei ihnen die Voraussetzungen einer unheilbaren Krankheit und des

110 Schreiber, NStZ 1986, S. 337, 340; ders., FS Hanack, S. 735, 736; Oduncu/Eisenmenger, MedR 2002, S. 327, 329.

111 Kutzer, MedR 2001, S. 77, 78; ders., GS Schlüchter, S. 346, 357; Körtner, ZME 2002, S. 15, 18; Oduncu/Eisenmenger, MedR 2002, S. 327.

112 Vgl. zu ähnlichen Fallgestaltungen Wolfslast, Brauneck-EG, S. 473, 492. Das letzte Beispiel zeigt erneut die Klugheit der Installierung einer solche Entscheidungen aus objektiver Sicht begutachtenden Kontrollinstanz in Form des Vormundschaftsgerichts, vgl. oben bei Fn. $111 \mathrm{ff}$. 
unerträglichen Leidens nicht gegeben sind. Führt man sich dies vor Augen, verliert das Argument auf der einen Seite einiges von seiner Wirkungskraft.

Auf der anderen Seite lenkt es den Blick auf Personen, die nicht den genannten Kriterien unterfallen, denen jedoch der Wille zum Weiterleben fehlt. ${ }^{113}$ Die Zahl dieser Personen dürfte in nicht ferner Zukunft aufgrund des Zusammenspiels verschiedener gesellschaftlicher Entwicklungen erheblich anwachsen. Zum einen machen es die Fortschritte der Medizin immer wahrscheinlicher, ein hohes Lebensalter ohne schwerwiegende Leiden und nur mit den quasi »natürlichen« Einschränkungen zu erreichen. Die Aussicht eines einsamen Sterbens nach einem in den letzten Lebensjahrzehnten sozial isolierten Dasein ergibt gemeinsam mit einer subjektiv empfundenen Abwertung des Alterns als Folge der in der Gesellschaft verbreiteten Glorifizierung von allem Jugendlichen eine Mischung, die schnell zu dem Wunsch nach Beendigung des eigenen Lebens führen kann. Angesichts des Umstandes, dass die Unerträglichkeit eines Leidens subjektiv empfunden wird und auch ein körperlich und geistig im wesentlichen gesunder Mensch sein Dasein als unerträglich empfinden kann, stellt sich die Frage, warum für Patienten mit infauster Prognose ohne Behandlungsperspektive andere Kriterien gelten sollen als für diese Menschen. An dieser Stelle kommt die Frage in unser Blickfeld, ob eine Gesellschaft es Dritten gestatten soll, sich auf Bitten des Patienten zum Herrscher über dessen Todesprozess aufzuschwingen. Die Alternative wäre, den gegenwärtigen Zustand zu belassen und mittels einer im Kern paternalistischen Rechtsnorm die Gesellschaft und auch die Todeswilligen selbst zu schützen.

\section{Ergebnis und Ausblick}

\section{Differenzierung nach der Hilflosigkeit des um den Tod Bittenden}

Jedenfalls für solche Personen, die körperlich in der Lage sind, ihrem als unerträglich empfundenen Zustand selbst ein Ende zu setzen, sollte dieses Verbot aufrechterhalten werden. Derjenige, der die Selbsttötung eigenhändig vollzieht, hat auch bis zuletzt die Möglichkeit, seinen Willen noch zu ändern und ein Weiterhandeln zu unterlassen, während derjenige, der das Geschehen in fremde Hände legt, eine solche Rückzugsmöglichkeit nicht mehr besitzt. ${ }^{114}$ Die grundsätzliche Anerkennung eines Selbstbestimmungsrechts über das eigene Leben setzt angesichts der staatlichen Verpflichtung zum Schutz des Lebens in besonderer Weise voraus, dass die Entscheidung autonom und endgültig getroffen wurde. ${ }^{115}$ Derjenige, der trotz einer physischen Möglichkeit psychisch nicht in der Lage ist, sie selbst $\mathrm{zu}$ vollziehen, sondern sie einem anderen aufbürdet, gibt genügend Anlass, an der Autonomie seines Sterbewillens zu zweifeln, um solche Handlungen von Seiten des Rechts weiterhin als verboten einzustufen. ${ }^{116}$ Im Gegenteil erscheint es ethisch verwerflich, eine solche Entscheidungslast

113 Die Problematik dieser Gruppe erkennt auch Tak, ZStW 113 (2001), S. 905, $921 \mathrm{f}$.

114 Bottke, in: Lebensverlängerung (o. Fn. 18), S. 35, $109 \mathrm{f}$.

115 Verrel, JZ 1996, S. 224, 226.

116 Ebenso Verrel, JZ 1996, S. 224, 226, a.A. allerdings Gutmann, Ethik Med 2002, S. 170, 173 f. 
dem Lebenspartner, dem eigenen Kind oder dem Hausarzt aufzubürden, während man sich selbst zu einem solchen Schritt nicht entschließen kann. Die von dem Strafrechtler Scheffler provokant gestellte Frage, ob man sich den Verzicht darauf, die Hilfe eines anderen anzunehmen, mit der Begründung abnötigen lassen müsse, man könne sein Ziel auch selbst erreichen ${ }^{117}$, ist also in eben dieser Klarheit zu bejahen, es handelt sich um die Kehrseite des die personale Autonomie sichernden Prinzips der Eigenverantwortung.

Anders stellt sich die Situation in Fällen dar, in denen der tödlich kranke und glaubhaft sterbewillige Patient körperlich nicht mehr in der Lage ist, seinem Leiden selbst ein Ende zu setzen. Für solche Situationen sollte in $\S 216$ StGB ein Ausnahmetatbestand geschaffen werden, der auch die aktive Tötung solcher Patienten erlaubt, sofern die Möglichkeiten der Palliativmedizin nicht ausreichen, um das Leiden des Patienten zu lindern und sofern Verfahrensgarantien geschaffen worden sind, die mittels einer Formalisierung des Verfahrens einen möglichen Missbrauch zurückdrängen. ${ }^{118}$ Eine solche Vorgehensweise ermöglicht es, die bereits vielfach durchbrochenen Dogmen der Unantastbarkeit des menschlichen Lebens und des Verbots der aktiven Sterbehilfe nicht auf Kosten der schwächsten Gruppe der am Lebensende stehenden Menschen durchhalten zu müssen. Sie ist auch nicht das Einfallstor für weitergehende Lockerungen im Bereich der Ethik des Lebensschutzes, die zu Angstzuständen bei älteren Menschen führen müssten, sondern könnte im Gegenteil eine geradezu befriedende Wirkung haben, indem einem Leidenden der Prozess seines Sterbens durch den Gedanken erleichtert werden kann, dass er als letztes Mittel das Thema einer Lebensbeendigung ansprechen könnte, ohne die damit konfrontierte Person in einen tiefen Konflikt zwischen Mitmenschlichkeit und Rechtstreue zu stürzen. ${ }^{119}$

Es handelt sich wohl um die schwerste Entscheidung, die an einen Menschen herangetragen werden kann, einem geliebten bzw. anvertrauten Menschen zum Tode zu verhelfen. Diese Entscheidung stellt den Betroffenen vor eine tiefe Zerreißprobe und wird oftmals unabhängig von ihrem Ausgang zu späterer Zeit auf emotionaler Ebene tief bedauert. Die Frage ist, ob dieser existentielle Konflikt dadurch noch verstärkt werden muss, dass keinerlei Möglichkeiten belassen werden, in rechtlicher Hinsicht nicht als Konsequenz einer solchen Entscheidung belangt zu werden. ${ }^{120}$ Eine Beschränkung des Rechts auf Sicherung von Rahmenbedingungen mittels Schaffung nachträglicher Meldepflichten oder sonstiger prozessualer Voraussetzungen erscheint angezeigt.

117 In: Joerden (Hrsg.); Der Mensch und seine Behandlung in der Medizin, S. 249, 261.

118 Im Ergebnis wie hier Kutzer, ZRP 1997, S. 117, 119; ebenso ders., MedR 2001, S. 77, 78; Birnbacher, Rechtsphilosophische Hefte 1998, S. 75, 84.; Wolfslast, Brauneck-EG, S. 473. 494; Otto, ZfL 2002, S. 42, 48; zur verfassungsrechtlichen Legitimation solcher Ausnahmetatbestände Hufen, NJW 2001, S. 849, 855 mwN.

119 Vgl. auch Schreiber, NStZ 1986, S. 337, 345; Wolfslast, Brauneck-EG, S. 473, 494; Birnbacher, RpH 1998, S. 75, 81f.

120 Ebenso Arthur Kaufmann, FS Roxin, S. 841, 852. 


\section{2. Überprüfung des Ergebnisses: Vereinbarkeit mit dem allgemeinen Tötungsverbot?}

Das gefundene Ergebnis geht nicht nur über das gegenwärtig von den Normen des Rechts Erlaubte hinaus, sondern könnte zudem mit einer ethischen Fundamentalnorm, dem Tötungsverbot, unvereinbar sein. Das Tötungsverbot wird im christlich-abendländischen Kulturkreis traditionell in metaphysischer Weise auf die Gottebenbildlichkeit des Menschen und die daraus herleitbare Unantastbarkeit menschlichen Daseins zurückgeführt. ${ }^{121}$ Indem der Mensch das Leben von Gott empfangen hat, liegt es nicht in seiner oder eines Dritten Hand, ihm ein Ende zu setzen. Der Einstellung, mit der Vornahme der Euthanasie solle nicht lediglich das Leben, sondern primär ein Leidenszustand des Kranken beendet werden, wird entgegengehalten, dass auf diese Weise eine ethisch unwerte Gleichsetzung des Lebens mit der Krankheit vorgenommen werde. $^{122}$ Im Zuge von Säkularisierung und Pluralisierung der Lebens- und Wertvorstellungen werden jedoch religiös-metaphysische Begründungen des Tötungsverbots immer weniger akzeptiert. Das Tötungsverbot wird vermehrt nach utilitaristischen Gesichtspunkten auf das Interesse des Betroffenen gestützt. ${ }^{123}$ Unabhängig hiervon erscheint es den vielfältigen, am Ende des menschlichen Lebens auftretenden Leidenssituationen jedenfalls nicht gerecht zu werden, wenn Vertreter der »sanctity of life «Doktrin das Leben als absoluten, von den Präferenzen des Betroffenen abzutrennender Höchstwert gesehen wird, wenn gleichzeitig kein Anstoß daran genommen wird, zur Wahrung körperlicher Unversehrtheit oder materieller Güter als letztes Mittel das Leben eines Angreifers zu nehmen. ${ }^{124}$ Ist demjenigen, der mit radikalen und vom Recht gestatteten Mitteln sein Eigentum verteidigt, kein Vorwurf zu machen, so kann der einen geliebten oder unter seiner Obhut stehenden Menschen auf dessen Flehen hin Erlösende, der damit eine Entscheidung trifft, die ihn in seinem späteren Leben vielleicht nie wieder loslässt, nicht schlechter gestellt werden.

Eine weitere Inkonsistenz der gegenwärtigen Debatte besteht darin, dass sie Elemente der sanctity of life - Doktrin und den Gedanken des Selbstbestimmungsrechts des Patienten ohne Rücksicht auf Friktionen nebeneinander stellt. So muss dem Wunsch nach Sterbenlassen, wie unvernünftig er auch sei, unter allen Umständen

121 Vgl. statt aller Eibach, ZME 42 (1996), S. 312, $316 f$.

122 Eibach, ZME 42 (1996), S. 312, 317.

123 Vgl. Singer, Rethinking Life and Death, S. 74f., 219ff.; Hoerster, Sterbehilfe im säkularen Staat, S. 24 u. pass. Die Diskussionen in Bezug auf Menschenwürde und Personalität, die diese neue Sichtweise aufgeworfen hat, können hier nicht behandelt werden (vgl. dazu statt aller Merkel, Früheuthanasie, S. 391ff. u. pass.; ablehnend insb. Eibach, MedR 2002, S. 123ff.). Ein Anschluss an ihre Konsequenzen im Hinblick auf das Lebensrecht schwerstgeschädigter neugeborener oder komatöser Menschen soll ausdrücklich nicht erfolgen. Eine Unterscheidung zwischen biologisch-menschlichem Leben einerseits und personal-menschlichem Leben andererseits verkennt das Wesen der Autonomie als unabdingbaren Wesenszug des Menschen, die auch bei fehlender Möglichkeit zur Ausübung des Selbstbestimmungsrechts fortbesteht, vgl. dazu Beckmann, ZME 44 (1998), S. 143, 148ff.; Halliday/Witteck, JZ 2002, S. 752ff.

124 Im Ergebnis wie hier Duttge, NStZ 2001, S. 546, 547; Sternberg-Lieben, JZ 2002, S. 153, 155. 
Folge geleistet werden, dem Wunsch nach Getötetwerden, wie begründet er auch sei, darf unter keinen Umständen Folge geleistet werden. ${ }^{125}$ Dies führt dazu, dass das gesamte Gewicht der rechtlichen und auch der ethischen Differenzierung nach erlaubt und nicht erlaubt auf der brüchigen Unterscheidung von Tun und Unterlassen ruht, was angesichts der im ersten Teil dargestellten Entwicklungen nicht länger überzeugen kann. Die in $§ 216$ StGB niedergelegte Absolutheit des Tötungsverbotes hat ihren Sinn darin, vor übereilten Handlungen in Krisensituationen zu schützen. Sie verliert ihre Legitimation jedoch dann, wenn die Sterbensinteressen des Menschen so fundamentale Bedeutung erlangen, dass ein absolutes Tötungsverbot als inhumaner Zwang zum Weiterleben wirkt. ${ }^{126}$

Auch von Seiten der Gegner einer aktiven Sterbehilfe muss folglich akzeptiert werden, dass es Fälle gibt, in denen auch und gerade aus ethischen Erwägungen eine absolute Geltung des Tötungsverbots einem wahrhaft humanen Handeln im Wege stehen kann und damit kontraproduktiv wirkt. In diesen seltenen Fällen muss das Interesse der Gesellschaft an einem wirksamen Lebensschutz hinter dem Interesse des Patienten an der Beendigung des Lebens zurückstehen. Wichtig ist dabei jedoch, dass das Mitleidsmotiv zwar eine notwendige, aber keine hinreichende Bedingung der teilweisen Legalisierung aktiver Euthanasie ist. Eine sorgfältige Prüfung der Ernsthaftigkeit des Wunsches und der Aufrichtigkeit der Motive des Handelnden von Seiten einer unabhängigen Institution ist ebenso Voraussetzung wie die hier vorgeschlagene strikte Beschränkung des Anwendungsbereichs auf hoffentlich immer seltener werdende Fälle.

Die hier vertretene Position kann gleich von zwei Seiten angegriffen werden, indem man ihr einerseits vorhält, den Unterschied zwischen dem Sterbenlassen und dem aktiven Töten eines Menschen zu negieren und durch eine partielle Gleichsetzung dieser Vorgehensweisen Tür und Tor für ein lebensverachtendes Rechtssystem zu öffnen. Andererseits kann man Inkonsequenz attestieren, da die Autonomie des Patienten zwar als Leitfigur der Entscheidungen am Lebensende eingestuft wird, diesem Wert aber zugleich eine Grenze durch staatlichen Paternalismus gezogen wird, wenn es um die aktive Tötung eines des Lebens Überdrüssigen geht. Dem kann nur entgegengehalten werden, dass es die Humanität zuweilen gebietet, Handlungen trotz einer gewissen Missbrauchsgefahr durchzuführen und dass es den Lebensschutz nicht schwächt, sondern sogar stärkt, wenn mit dem hier eingeschlagenen Weg die Entscheidung über das Sterbenlassen eines Menschen aus den »Hinterzimmern « herausgezogen wird. Gegenüber Kritikern, die eine liberalere Position als die hier eingenommene vertreten, ist darauf zu bestehen, dass es kein Ausdruck der Autonomie des Menschen ist, wenn er eine Entscheidung, die er von seinen körperlichen Möglichkeiten wie auch von Rechts wegen selbst durchführen könnte, einem Dritten aufbürdet. Das Recht auf Selbstbestimmung umschließt wenigstens in diesen Fällen auch die Pflicht, die Beendigung des eigenen Lebens nicht einer dritten Person zu überlassen. Das Selbstbestimmungsrecht des Einzelnen findet seine Grenze dort, wo andere zu Handlangern eigener

$125 \mathrm{Vgl}$. hierzu auch Merkel, Früheuthanasie, S. 419f.

126 Ebenso Merkel, Früheuthanasie, S. 421ff. 
Absichten gemacht werden, für die das Individuum selbst noch Verantwortung übernehmen kann.

Einer gesetzlichen Ausnahmeregelung wird entgegengehalten, neue Gesetze schadeten in diesem sensiblen Bereich mehr als sie nutzen. ${ }^{127}$ Jedoch ist neben der Erleichterung, die eine gesetzliche Klarstellung der Grenzen aktiver Sterbehilfe für Ärzte und Pflegepersonal bedeuten würde ${ }^{128}$, auf den Gewinn an Rationalität der Entscheidungsfindung hinzuweisen, der einer Tendenz zu informellem und missbräuchlichem Verhalten sogar entgegenwirken könnte und so wesentlich mehr nützen als schaden würde. Die Installierung einer gesetzlichen Regelung schließt weiterhin nicht aus, dass dem Arzt Handlungsweisen erlaubt werden, die ihm seine Berufsethik untersagt. Die notwendige Wechselbezüglichkeit von Rechtsnormen, allgemeiner Ethik und Standesethik gestattet ein solches Auseinanderfallen von Vorgaben durchaus. Gerade diese unterschiedlichen »Signale«, die der vor einer schweren Entscheidung stehende Arzt empfängt, können hilfreich sein, um sich jeglicher Aspekte dieser Entscheidung bewusst zu werden und so zu einem ethisch für ihn selbst und die Mehrzahl der Bevölkerung akzeptablen Ergebnis zu gelangen. ${ }^{129}$ Dies scheint bereits das Maximum dessen zu sein, was mittels rechtlicher Vorgaben dem einzelnen Menschen zu seiner Entscheidung an die Hand gegeben werden kann.
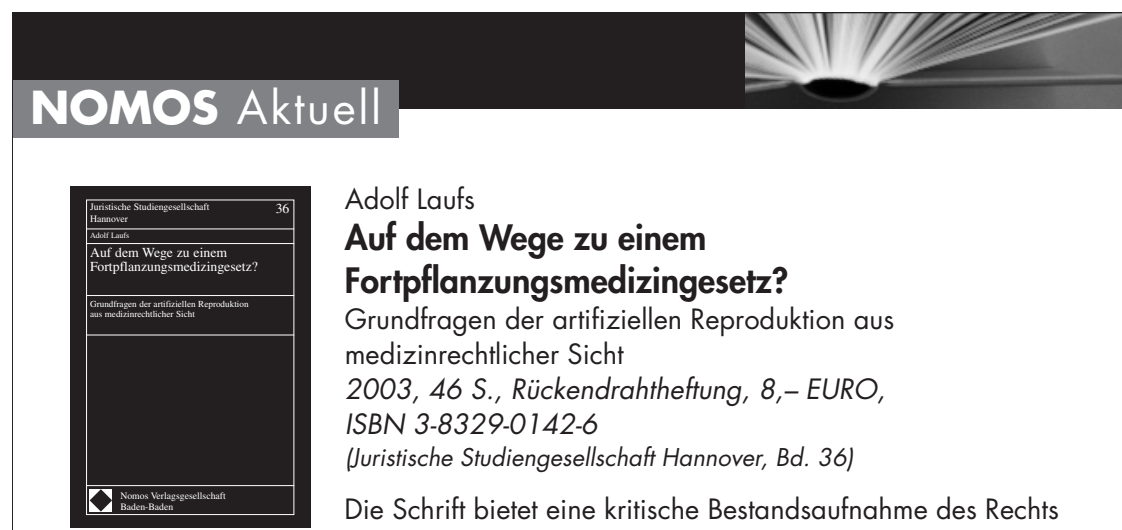

Adolf Laufs

Auf dem Wege zu einem Fortpflanzungsmedizingesetz?

Grundfragen der artifiziellen Reproduktion aus medizinrechtlicher Sicht 2003, 46 S., Rückendrahtheftung, 8,-EURO, ISBN 3-8329-0142-6

(Juristische Studiengesellschaft Hannover, Bd. 36)

Die Schrift bietet eine kritische Bestandsaufnahme des Rechts der künstlichen Fortpflanzung. Eine Neuordnung unter Preisgabe der Gewährleistungen des Embryonenschutzgesetzes lehnt sie ab.

NOMOS Verlagsgesellschaft • Baden-Baden

127 Kutzer, ZRP 1997, S. 117, 119; kritisch gegenüber gesetzlichen Regelungen in diesem Bereich auch Tröndle, ZStW 99 (1987), S. 25, 41, 47; vgl. auch Otto, Strafrecht BT, § 6 RN 33f., der die hier angesprochenen Fälle über die Regelungen des rechtfertigenden Notstandes aus dem Bereich der Strafbarkeit ausschließen will.

128 Vgl. hierzu Schreiber, NStZ 1986, S. 337, 343.

129 Vgl. Eser, JZ 1986, S. 786, 789. 